\title{
WestVirginiaUniversity
}

THE RESEARCH REPOSITORY @ WVU

Graduate Theses, Dissertations, and Problem Reports

2021

\section{IoT Malicious Traffic Classification Using Machine Learning}

Michael Austin

maa0075@mix.wvu.edu

Follow this and additional works at: https://researchrepository.wvu.edu/etd

Part of the Other Computer Engineering Commons

\section{Recommended Citation}

Austin, Michael, "IoT Malicious Traffic Classification Using Machine Learning" (2021). Graduate Theses, Dissertations, and Problem Reports. 8024.

https://researchrepository.wvu.edu/etd/8024

This Problem/Project Report is protected by copyright and/or related rights. It has been brought to you by the The Research Repository @WVU with permission from the rights-holder(s). You are free to use this Problem/Project Report in any way that is permitted by the copyright and related rights legislation that applies to your use. For other uses you must obtain permission from the rights-holder(s) directly, unless additional rights are indicated by a Creative Commons license in the record and/ or on the work itself. This Problem/Project Report has been accepted for inclusion in WVU Graduate Theses, Dissertations, and Problem Reports collection by an authorized administrator of The Research Repository @ WVU. For more information, please contact researchrepository@mail.wvu.edu. 


\title{
IoT Malicious Traffic Classification Using Machine Learning \\ Michael Austin
}

\author{
Problem Report \\ submitted to the Benjamin M. Statler College of Engineering and Mineral Resources at \\ West Virginia University \\ in partial fulfillment of the requirements \\ for the degree of \\ Master of Science with Area of Emphasis in Cybersecurity \\ Committee Chair, Katerina Goseva-Popstojanova Ph.D. \\ Roy Nutter, Ph.D. \\ Thomas Devine, Ph.D. \\ Lane Department of Computer Science and Electrical Engineering
}

\author{
Morgantown, West Virginia
}

2021

Keywords: IoT, malware, machine learning, random forest, SVM, Zeek

Copyright 2021 Michael Austin 


\section{Abstract \\ IoT Malicious Traffic Classification Using Machine Learning}

\section{Michael Austin}

Although desktops and laptops have historically composed the bulk of botnet nodes, Internet of Things (IoT) devices have become more recent targets. Lightbulbs, outdoor cameras, watches, and many other small items are connected to WiFi and each other; and few have well-developed security or hardening. Research on botnets typically leverages honeypots, PCAPs, and network traffic analysis tools to develop detection models. The research questions addressed in this Problem Report are: (1) What machine learning algorithm performs the best in a binary classification task for a representative dataset of malicious and benign IoT traffic; and (2) What features have the most predictive power? This research showed that the best performing algorithms were Random Forest with accuracy of $97.45 \%$ and $F_{1}$ score of $97.39 \%$; and the Linear SVM with a recall score of $99.90 \%$. The most important features for the classification were: time of day, history, protocol, and count of origin bytes sent. Of these, time of day and volume of traffic coming from the same IP addresses are consistent for port scanning, infection, and distributed denial of service attacks. 


\section{List of Figures}

1.1 Timeline of IoT malware families through 2018, per Costin and Zaddach . . 3

1.2 Bashlite DDoS mitigation and scanner for embedded systems . . . . . . . . 4

1.3 Hide N' Seek hard-coded P2P IP addresses from [38] . . . . . . . . . . . . . 8

1.4 Tomato default credential Shodan search . . . . . . . . . . . . . . . . . 9

1.5 Muhstik IRC C\&C . . . . . . . . . . . . . . . . . . . . . . . . . 9

1.6 Shodan default passwords router search . . . . . . . . . . . . . . . . . . . 10

3.1 Wireshark frame with Zeek capture elements: Part 1 . . . . . . . . . 15

3.2 Wireshark frame with Zeek capture elements: Part 2 . . . . . . . . . 16

3.3 Zeek conn.log example . . . . . . . . . . . . . . . . . 17

5.1 Full dataset breakdown after subsampling malicious category . . . . . . . . . 28

5.2 Detailed breakdown of malicious label subgroups after subsampling . . . . . 29

5.3 Protocol count of malicious and benign traffic . . . . . . . . . . . . 30

5.4 Box plot of performance metrics . . . . . . . . . . . . . . . . 32

5.5 Random Forest feature importance, measured by Gini impurity . . . . . . . 33

5.6 Precision-recall curve for linear SVM . . . . . . . . . . . . . . . . . 34 


\section{List of Tables}

1.1 Hajime architecture-specific functions and their vulnerable services $\ldots$. . . 7

3.1 Zeek conn.log features (bolded features used in this problem report) . . . . . 18

3.2 Zeek history reference with description . . . . . . . . . . . . . 19

3.3 Number of packets in original packet captures, Zeek flows, and malware in scenarios used . . . . . . . . . . . . . . . . . . . 19

3.4 Detailed labels of the malicious activity of each flow in the IoT-23 Zeek logs 20

4.1 The metrics used to evaluate performance of the learners were computed from values from the confusion matrix: . . . . . . . . . . . . . . . 24

5.1 Statistics of models accuracy . . . . . . . . . . . . . . . . . . 31

5.2 Statistics of models F-1 scores . . . . . . . . . . . . . . . . . . . 31

5.3 Statistics of models recall . . . . . . . . . . . . . . . . . . . . 31

5.4 Statistics of models precision . . . . . . . . . . . . . . . 32 


\section{Acronyms}

AFC Apple File Conduit. 2

ANN Artificial Neural Network. 13

API Application Programming Interface. 4

BYOD Bring Your Own Device. 2

C\&C Command and Control. 5, 7, 8

CVE Common Vulnerabilities and Exposures. 6

DDoS Distributed Denial of Service. 3-5, 7, 8, 27, 34

DHT Distributed Hash Table. 6

IoT Internet of Things. 1-3, 5, 8, 9, 11, 14, 36, 38

ITU International Telecommunications Union. 1

NB Naïve Bayes. 22, 23

P2P Peer-to-Peer. 6, 8

RF Random Forest. 13

SGD Stochastic Gradient Descent. 23

SVM Support Vector Machine. 13, 22, 23 


\section{Dedication}

This body of work is dedicated to my mother, grandmother, and advisor. For all of their patience I have exhausted, time I have used, and generosity I have benefited from was not in vain. I would also like to thank Brian Powell for giving me the opportunity to work as a teaching assistant in the department and for taking a chance on me. Your support changed my life for the better and gave me new perspectives I will carry with me into my next endeavors. 


\section{Acknowledgements}

I would like to thank Drs. David Martinelli and Diana Knott Martinelli for their continued encouragement and friendship, and for introducing me to the department as a whole. I would also like to thank Drs. Roy Nutter and Thomas Devine for serving on my committee, working with me over the years, and forcing me to leave my comfort-zone in pursuit of more challenging and satisfying work. Although many more people contributed to my growth in computer science, these are the primary drivers. Thank you. 


\section{Contents}

1 Introduction $\quad 1$

1.1 IoT Devices . . . . . . . . . . . . . . . . . . . 1

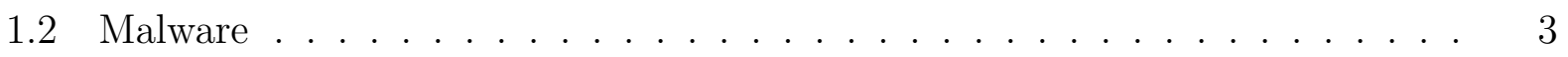

1.2.1 Gagfyt (Bashlite) . . . . . . . . . . . . . . 3

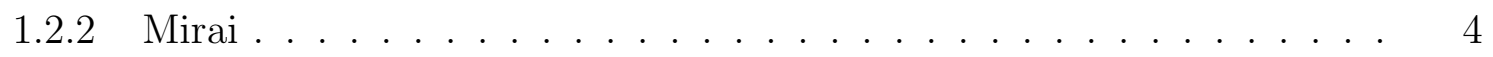

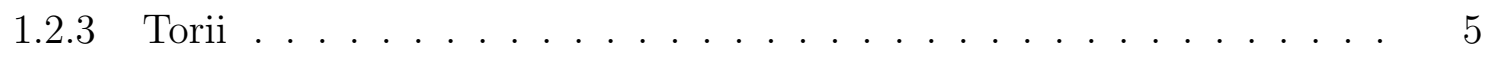

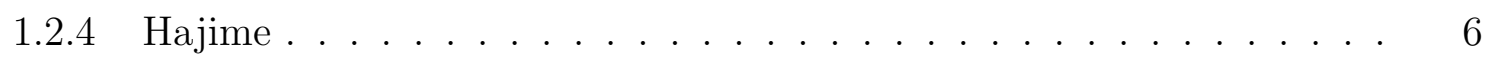

1.2.5 Hakai ........................ 7

1.2.6 Hide N'Seek . . . . . . . . . . . . . . . . . . 7

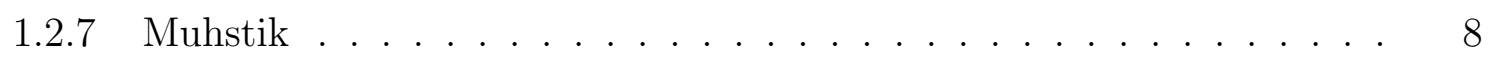

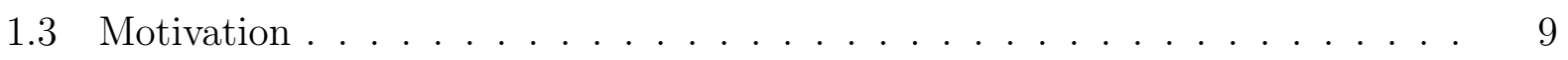

1.4 Research Questions ....................... . 10

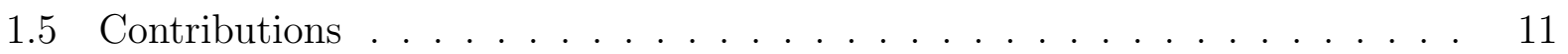

2 Related Work $\quad 12$

3 Description of the dataset and features $\quad 14$

3.1 IoT-23 Dataset . . . . . . . . . . . . . . . . . . . . 14

3.2 Zeek Connection Log Features . . . . . . . . . . . . . . . . . 17

4 Machine Learning $\quad 21$

4.1 Approach . . . . . . . . . . . . . . . . . . . . . 21

4.1.1 Random Forest . . . . . . . . . . . . . . . . . . . 22

4.1 .2 Naïve Bayes . . . . . . . . . . . . . . . . . . . . . . . 22

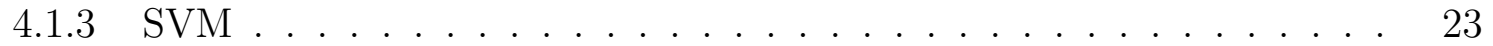

4.1.4 Stochastic Gradient Descent . . . . . . . . . . . . . . . . . . . 23

4.2 Performance Metrics . . . . . . . . . . . . . . . . . . . . . . . . . . 24 


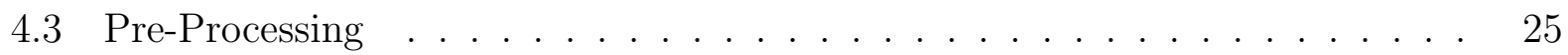

4.4 Feature Inclusion / Exclusion Criteria . . . . . . . . . . . . . . 26

5 Results of Machine Learning $\quad 27$

5.1 Analysis of the Dataset . . . . . . . . . . . . . . . . . 27

5.2 RQ1: What learner performs the best in terms of classification accuracy, recall, and precision for this IoT traffic dataset? . . . . . . . . . . . 30

5.3 RQ2: What are the features with the best predictive power for classification tasks? . . . . . . . . . . . . . . . . . . . . . . 32

5.4 Discussion of the Main Findings . . . . . . . . . . . . . . . . 34

6 Threats to Validity 35

6.1 Internal Threats to Validity . . . . . . . . . . . . . . . . 35

6.2 External Threats to Validity . . . . . . . . . . . . . . . . . . 36

6.3 Construct Threats to Validity . . . . . . . . . . . . . . 36

6.4 Conclusion Threats to Validity . . . . . . . . . . . . . . . . . . . 37

$\begin{array}{lll}7 & \text { Concluding Remarks } & 38\end{array}$ 


\section{Chapter 1}

\section{Introduction}

Our appliances, healthcare instruments, economy, and critical infrastructure rely on the availability, privacy, and integrity of computers to provide life-sustaining services and allow us to thrive as a society. As more devices connect to the internet and each other, the value of attacking them rises, too. Researchers calculated the costs of cybercrime to US entities (including intellectual property theft, data breaches of sensitive customer information, recovery, and lost productivity) was $\$ 385$ billion in $2012[27,16]$. According to McAfee researchers, this damage is now a $\$ 945$ billion anchor for the economy [47]. Cybercrime includes Distributed Denial of Service (DDoS), ransomware, spam, intellectual property and identity theft, and data breaches, among other illicit activity. When critical infrastructure such as power plants, hospitals, and financial services are rendered inoperable, these costs may include people's lives $[47,27]$. For these reasons, the importance of cybersecurity cannot be overstated.

\section{$1.1 \quad$ IoT Devices}

Internet of Things (IoT) devices is a nebulous term now applied to most appliances we are familiar with: thermostats, lightbulbs, phones, routers, printers, and entertainment systems. The International Telecommunications Union (ITU) is an international standards organization interested in the connectivity of communications networks, RF spectra, and satellite orbits [51]. They characterize IoT devices as industrial products that assume "smart" capabilities through sensors, that can be queried and interacted with remotely, and facilitate communication and connectivity between people and things [23]. For the purposes of this research, we will lean on this definition. 
The value created by these devices and services stems from the accessibility, convenience, and enhancements of combining mundane physical objects with IT-based digital services [54]. An example of this is an average outdoor motion-detecting light. When combined with IoT services, detection of movement, facial recognition, and night-vision can be combined with cloud processing and mobile application notifications sent to the owner or a security company. A speaker with a microphone can also listen for the sound of gunshots or broken glass, as it is the case with the Amazon Echo. IoT devices are not restricted to small consumer appliances, as agriculture and manufacturing incorporate them to manage harvesters, drills, irrigation, and assorted dynamic processes remotely [54].

Although hackers previously targeted desktops, servers, and laptops, IoT devices gained popularity in the workplace with Bring Your Own Device (BYOD) culture and mobile devices provisioned by organizations [11]. Recent studies indicate these ubiquitous devices are a rich attack surface continuing to expand with the explosion of sensitive Big Data hosted on phones such as email, SMS, WiFi data, and the potential for data exfiltration [11]. Examples of mobile malware written with the intent of stealing credentials from iOS devices made insecure through a process known as "jail-breaking" are: AdThief, Unfold, and Mekie [11]. Jail-breaking in this context allows unfettered access to a phone's Apple File Conduit (AFC) and allows the user to install unsigned, unverified applications on their iOS device [11].

Costin and Zaddach expanded their study of IoT malware targets to include routers, printers, TVs, and an assortment of embedded devices [10]. After examining 60 IoT malware families with 48 unique vulnerabilities, they assembled a timeline, seen in Figure 1.1. According to them, generic source code precursors directed at embedded devices date back to 2001 [10]. Some of the obstacles related to studying IoT malware are: high platform heterogeneity, difficulty of emulating IoT devices, financial cost of scaling research, difficulty associated with removing malware (making reuse of assets challenging) [10, 53]. 


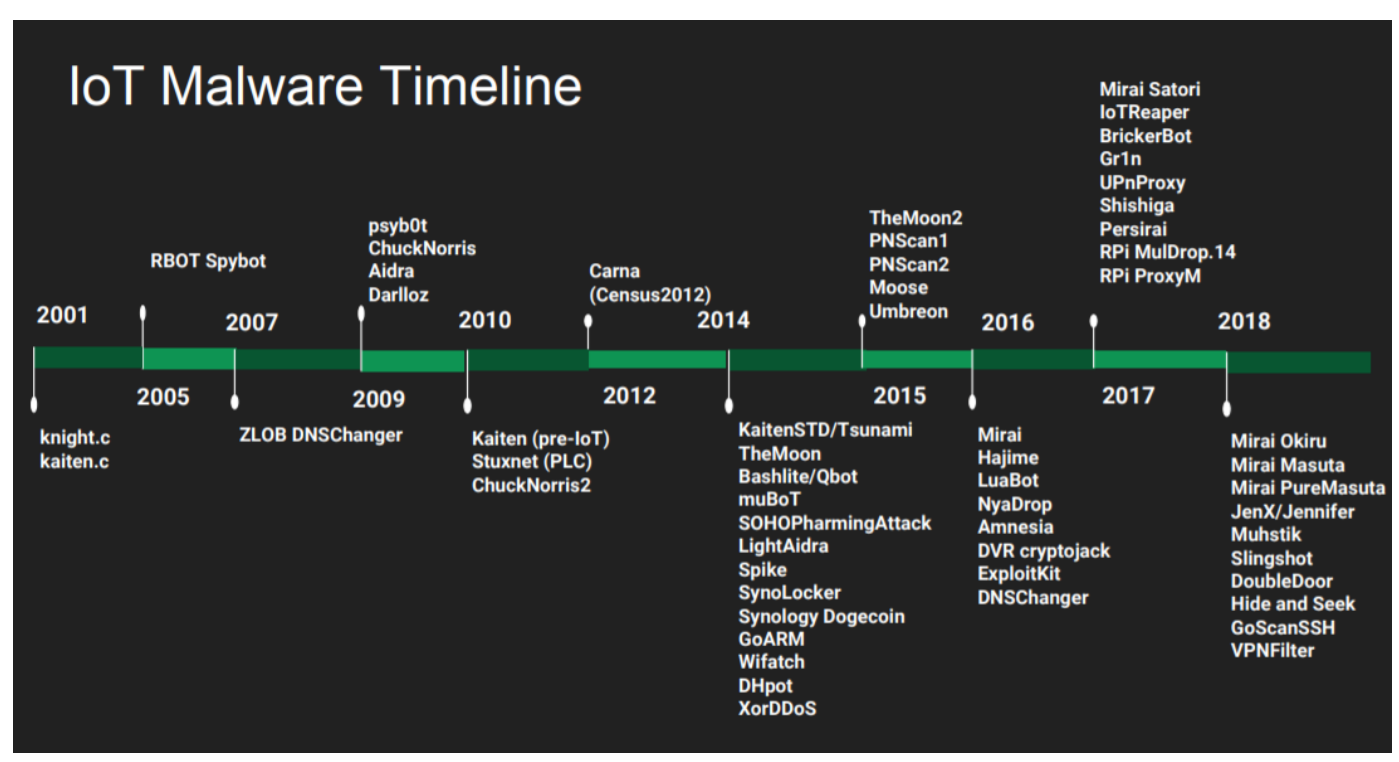

Figure 1.1: Timeline of IoT malware families through 2018, per Costin and Zaddach

\subsection{Malware}

Cybercriminals have expanded their target selection beyond routers and use infected IoT devices in a unified swarm of zombies, called a botnet, to perform DDoS attacks for black market customers [21]. The individual issuing commands to the botnet is known as a "botmaster." IoT devices can also be used to cripple a network until a ransom is paid, or surreptitiously mine crypto-currencies [21]. If an unsuspecting victim is an active member of a botnet, their IP address may be flagged and appear in blacklists, preventing access to some desirable parts of the internet or resulting in persistent, unintentional denials of service from legitimate hosts [21]. As of time of writing this, there are three main botnet progenitors: QBot (also known as Gafgyt or Bashlite), Kaiten, and Mirai [21, 1]. Gagfyt, Mirai, Torii, Hajime, Hakai, Hide N' Seek, and Muhstik were the malware used in this research and are described in greater detail, below.

\subsubsection{Gagfyt (Bashlite)}

Gagfyt (aka Bashlite, Torlus, Lizkebab) initially spread by exploiting shellshock vulnerabilities in Busybox on assorted devices in 2015 and is considered one of the progenitor IoT botnets [49]. Shellshock (CVE-2014-7169) is a Bash vulnerability (versions ; 4.3) that allows an attacker to execute code remotely by exporting shell functions to other bash instances via environment variables, including the CGI environment from web servers [49]. These exports 
take the form [49]:

env ENV_VAR_FN='() \{ <your function> $\}$; <attacker code here>'

Shellshock has also been used to execute denial of service attacks [49]. Exploits such as Shellshock are particularly pernicious because IoT devices historically have poor patch and security hygiene. It has since evolved to incorporate attacks against Universal Plug and Play (UPnP) APIs, cryptocurrency mining, backdoor payloads, a remote-code execution Metasploit module,DDoS functionality, and malware that competitively removes other malware on the victim machine [49]. After infection, Bashlite uses Telnet to perform reconnaissance and propagate. Bashlite commands can target embedded systems and, in some circumstances, bypass DDoS mitigation services, as seen in Figure 1.2 [49].

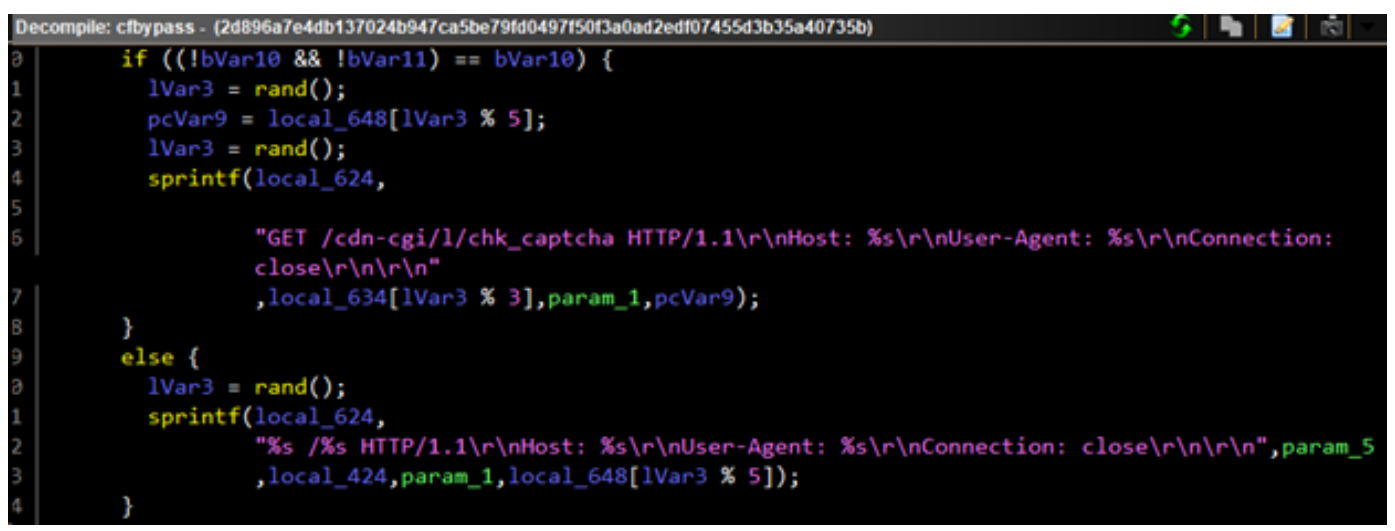

Figure 1.2: Bashlite DDoS mitigation and scanner for embedded systems

\subsubsection{Mirai}

Because several of the malware strains used in this problem report were Mirai variants, it is necessary to detail what makes Mirai worth analyzing. In October 2016, a massive DDoS attack against Dyn (a DNS infrastructure service provider) was launched from tens of millions of IP addresses [1]. This DDoS attack crippled data centers along the entire US east coast, Texas, Washington, and California [1,21]. A few months prior, Mirai was used to attack OVH, a French network provider for Minecraft, peaking at 800 Gbps in an identical fashion from 145,000 machines [1]. In both cases, a range of vulnerable IoT devices with no remote patching capability composed the botnet [1].

Variants of this malware cropped up following its source-code release on hackforums.net in 2016, including exploit support for a router autoconfiguration HTTP protocol vulnerability [1]. Victims are located by "statelessly," asynchronously scanning large blocks of IP addresses 
for an open Telnet port using a TCP SYN packet with a unique sequence number in the header $[1,22,52]$. After a suitable target is detected, Mirai attempts login via brute-forcing ten randomly selected pairs of the 62 credential tuples hard-coded [1]. Upon successful intrusion, credentials and IP address of the new node are sent to a specified report server and an asynchronous loader program infects the machine, downloads more architecturespecific malware, executes it, then begins obfuscation [1]. This proliferation occurs rapidly, as Mirai's doubling time is every 76 minutes [1]. Mirai will delete the downloaded binary files and rename the process to a pseudo-random alphanumeric string to camouflage its presence [1]. Once embedded, Mirai aggressively kills other processes bound to TCP/22 and TCP/23, including ones associated with other malware or Mirai variants [1,21].

\subsubsection{Torii}

Torii is named so because it emerges from Tor exit nodes to attack vulnerable IoT devices [2]. Although Torii brute-forces passwords in a dictionary attack similar to Mirai and attacks via telnet port 23, several notable features that make it stealthier and persistent distinguish it from its predecessor [2]. As far as recent research indicates, Torii does not engage in DDoS attacks, mine cryptocurrencies, or attack all devices on the network, as per usual $[2,19]$. Instead, Torii uses encrypted communication, data exfiltration stratagem, victim architecture discovery to download payloads specific to that device down to the endianness. The loader script attempts a variety of commands wget, ftpget, ftp, busybox wget, busybox ftpget to maximize the probability of a successful payload delivery [2]. To make analysis difficult, strings and parts of the second stage loader script were obfuscated by the author using an XOR-based encryption that is decrypted at runtime [2]. Evasion techniques include a one-minute sleep() after execution, symbol stripping from the executables, and pseudo-random process naming to avoid blacklist detection [2].

The second stage is an ELF binary downloaded to a pseudo-random destination based on a pre-defined file list of several options in the /tmp or / usr Linux directories [2]. Several methods are available to persist this second stage, including automatic execution via \.bashrc, a cronjob, systemd daemon, modification of SELinux Policy Management, or /etc/inittab [2]. Command and Control $(\mathrm{C} \& \mathrm{C})$ communication is also encrypted using the XOR cipher and a variety of domains to connect to: top.haletteompson.com, cloud.tillywirtz.com, and trade.andrewabendroth.com, which can be resolved via Google DNS at 8.8.8.8 to several other connected IP addresses [2]. The C\&C traffic uses port 443; however, it does not use TLS [2]. Furthermore, this traffic contains the data exfiltrated in an AES-128 encrypted 
package with an MD5 checksum to ensure lack of corruption, process ID, hostname, and path to the second stage ELF file, all MAC addresses found on the device, and distribution information found via the uname command [2]. These attributes indicate a level of sophistication that does not typify botnet malware.

\subsubsection{Hajime}

Although Hajime emulates infection tactics of Mirai such as the range of IP addresses blacklisted, several attributes differentiate this botnet from its predecessor [20, 1]. Hajime uses a decentralized, peer-to-peer (P2P) BitTorrent distributed hash table (DHT) to download malware to infected devices and update bots, utilizing a public key exchange in a custom protocol [20]. Furthermore, Hajime incorporates a wide range of access methods and targets a spectrum of CPU architectures in the ARM and MIPS families, as seen in Table 1.1 [20]. Immediately after establishing a beachhead on the victim machine, Hajime will block ports 23, 5358 (a telnet alternative), 5555, and 7547 using iptables to prevent reinfection and effectively "mark" its territory [20]. The botnet relies on two ELF executables (an atk and implant modules) and a config file to function [20].

The atk module is responsible for scanning and propagation. Blocked IP address ranges include private subnets, reserved and multicast ranges, IANA special use addresses, and some US federal subnets like the Department of Defense and Post Office [20]. Unusually, the botnet also excludes a handful of Middle Eastern and European subnets with particular exclusion of Dutch ISP ranges, indicating the author(s) is likely from the Netherlands [20]. Interesting CVEs associated with the atk module include CVE-2018-10561 and CVE-201810562, which bypass HTTP server authentication by passing "?images" to any login URL requests, allowing Hajime to execute shell code on Dasan GPON routers [13].

The implant package performs all P2P activity, including lookups for updates to the implant and atk modules, new orders, and seeding the config file of the day for the botnet [20]. Bots download files from each other through a modified version of the uTorrent Transfer Protocol, using well-established peers as a bootstrap that propagates malware payload updates rapidly, as is the case with an update to the atk module that included the Chimay-Red exploit for attacks on mipseb architectures circa March 25th, 2018 [20]. 
Table 1.1: Hajime architecture-specific functions and their vulnerable services

\begin{tabular}{|c|c|c|c|}
\hline Architecture & Port & Service & Method \\
\hline mipseb & $23,5358,7547,80$ & $\begin{array}{c}\text { Telnet, TR-064, } \\
\text { HTTP }\end{array}$ & $\begin{array}{c}\text { credentials, } \\
\text { CVE-2016-10372, } \\
\text { Chimay-Red, } \\
\text { CVE-2018-10561 } \\
\text { and } 62\end{array}$ \\
\hline mipsel & $23,5358,7547$ & Telnet, TR-064 & $\begin{array}{c}\text { credentials, } \\
\text { CVE-2016-10372 }\end{array}$ \\
\hline ARM7 & $23,5358,81$ & Telnet and HTTP & $\begin{array}{c}\text { credentials, } \\
\text { GoAhead-Webs, } \\
\text { Cross Web Server } \\
\text { RCE }\end{array}$ \\
\hline ARM6 & 23,5358 & Telnet & credentials \\
\hline ARM5 & $23,5358,9000$ & Telnet, MCTP & $\begin{array}{c}\text { credentials, } \\
\text { CVE-2015-4464 }\end{array}$ \\
\hline
\end{tabular}

\subsubsection{Hakai}

Hakai (Japanese for "destruction") is based on Gagfyt and leverages a critical vulnerability in Huawei HG352 routers that allows for remote code execution, after attackers send malicious packets to port 37215 (CVE-2017-17215) [35, 40, 34]. This botnet later exploited D-Link and Realtek routers through the HNAP protocol (CVE-2015-2051) in order to propagate [9]. An additional vulnerability Hakai adopted is CVE-2014-8361. The primary payload of the malware is a backdoor routine that can act as a dropper, DDoS attacker, and execute shell commands, according to TrendMicro [50]. Researchers identified a Telnet scanner, default password brute-forcing mechanism, and configuration table encryption that resemble those of Mirai, in addition to the zero-day vulnerabilities Hakai utilizes [50].

\subsubsection{Hide N' Seek}

Hide N' Seek (HNS) is a botnet with novel peer-to-peer (P2P) C\&C protocol use and infection methodology similar to Mirai [28, 38]. One of the plagiarized Mirai functions is the scanner which performs reconnaissance and exploits through ports 80 (HTTP), 8080 (HTTP), 2480 (OrientDB), 5984 (CouchDB), and 23 (Telnet) [38]. If these hard-coded exploits fail, a login 
brute-force attempt is made against the included dictionary of 250 credential sets, mainly composed of default passwords for devices such as routers [28, 38]. The second crucial method is the $\mathrm{C} \& \mathrm{C}$ and propagation mechanism which sends data about newly infected hosts, downloads and distributes additional binaries, and exfiltrates data from the host [28].

The botmaster uses the custom P2P protocol to retrieve the following information from the victim machine: configuration version number, reports a device to be scanned, send data from a file at a hashed location, send a file (such as a malicious binary) to an IP address at a port, and request/ receive the address and port of a peer [28, 38]. Sample code from the HNS peer network is seen in Figure 1.3 [38]. Each peer in the network has a SHA-512 hash of all the other files being distributed [5].

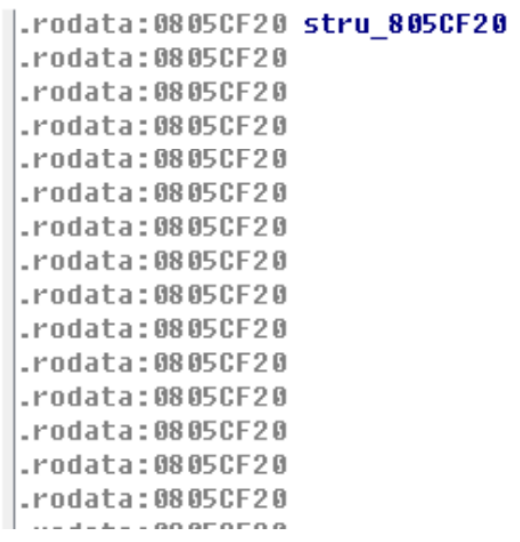

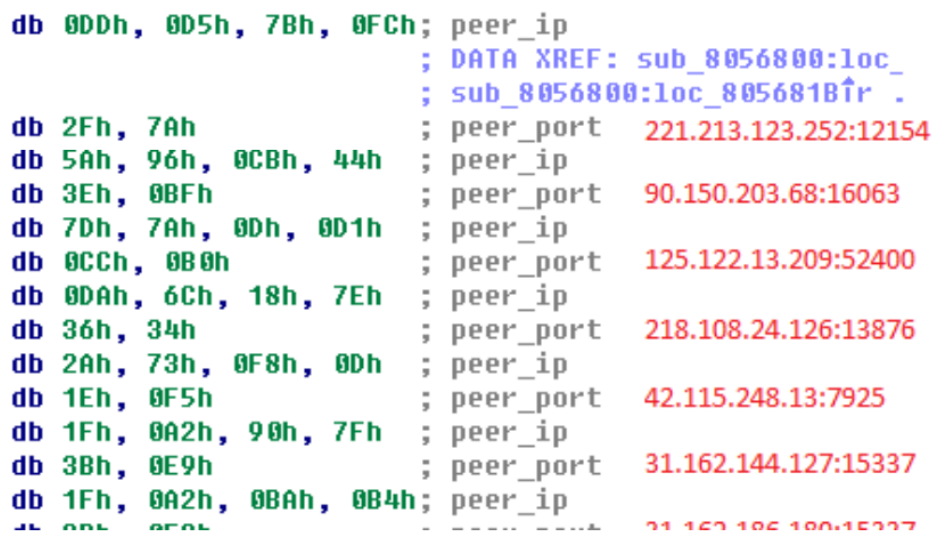

Figure 1.3: Hide N' Seek hard-coded P2P IP addresses from [38]

\subsubsection{Muhstik}

Since 2018, the Muhstik botnet has attacked DD-WRT and GPON IoT routers running services on such as WebLogic (CVE-2019-2725), WordPress (scanning ports 80 or 8080 and delivering a malicious file upload), and the Drupal content management system [35, 4]. In 2019, it added open-source Tomato router firmware to its repertoire of potential victims via port 8080 using brute-force methods [35]. After infection and proliferation, Muhstik leeches the resources of the host machine to mine cryptocurrencies and perform DDoS attacks via its IRC C\&C channel, seen in Figure 1.5 [35]. A quick Shodan search reveals some Tomato routers are still running default credentials and are vulnerable to the brute-force attempts seen in Figure 1.4 [42]. Another 1,500 were found when adding Tomato routers with NAS setups. 


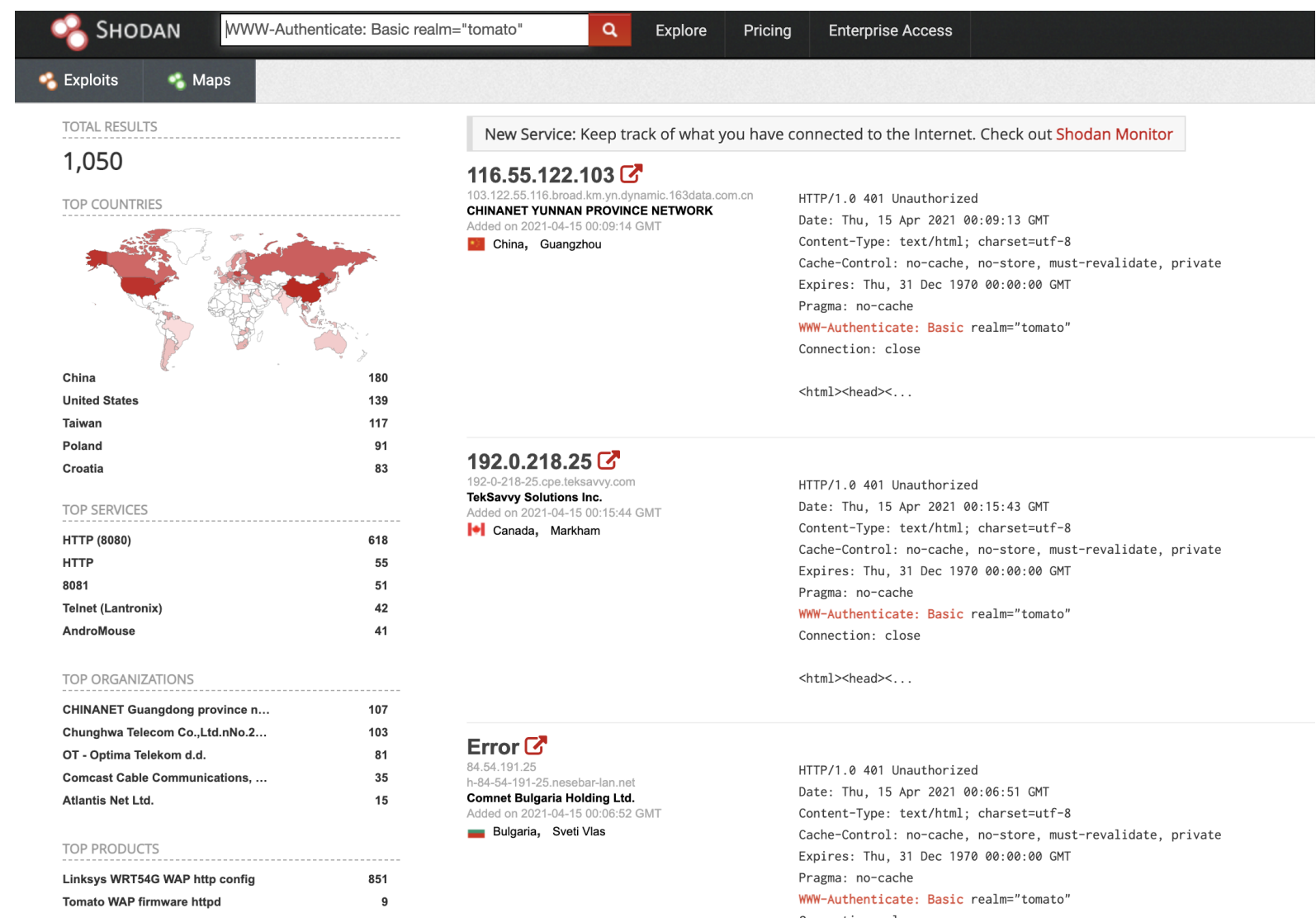

Figure 1.4: Tomato default credential Shodan search

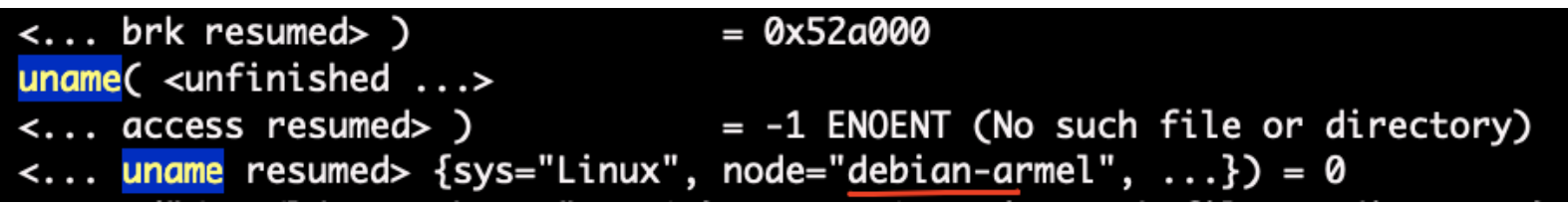

Figure 1.5: Muhstik IRC C\&C

\subsection{Motivation}

Even a cursory search on Shodan, a search engine for public-facing devices and the services running on them, reveals that tens of thousands of routers are exposed with default passwords still used Figure 1.6 [42]. Based on the attack vectors used in the malware explored in this body of work and the relatively weak security posture IoT devices maintain, there is clearly a need for better detection and mitigation. Furthermore, with the number of appliances, vehicles, and integrations with phones it is imperative to find the most accurate and robust models to detect malicious behavior and its future iterations. 


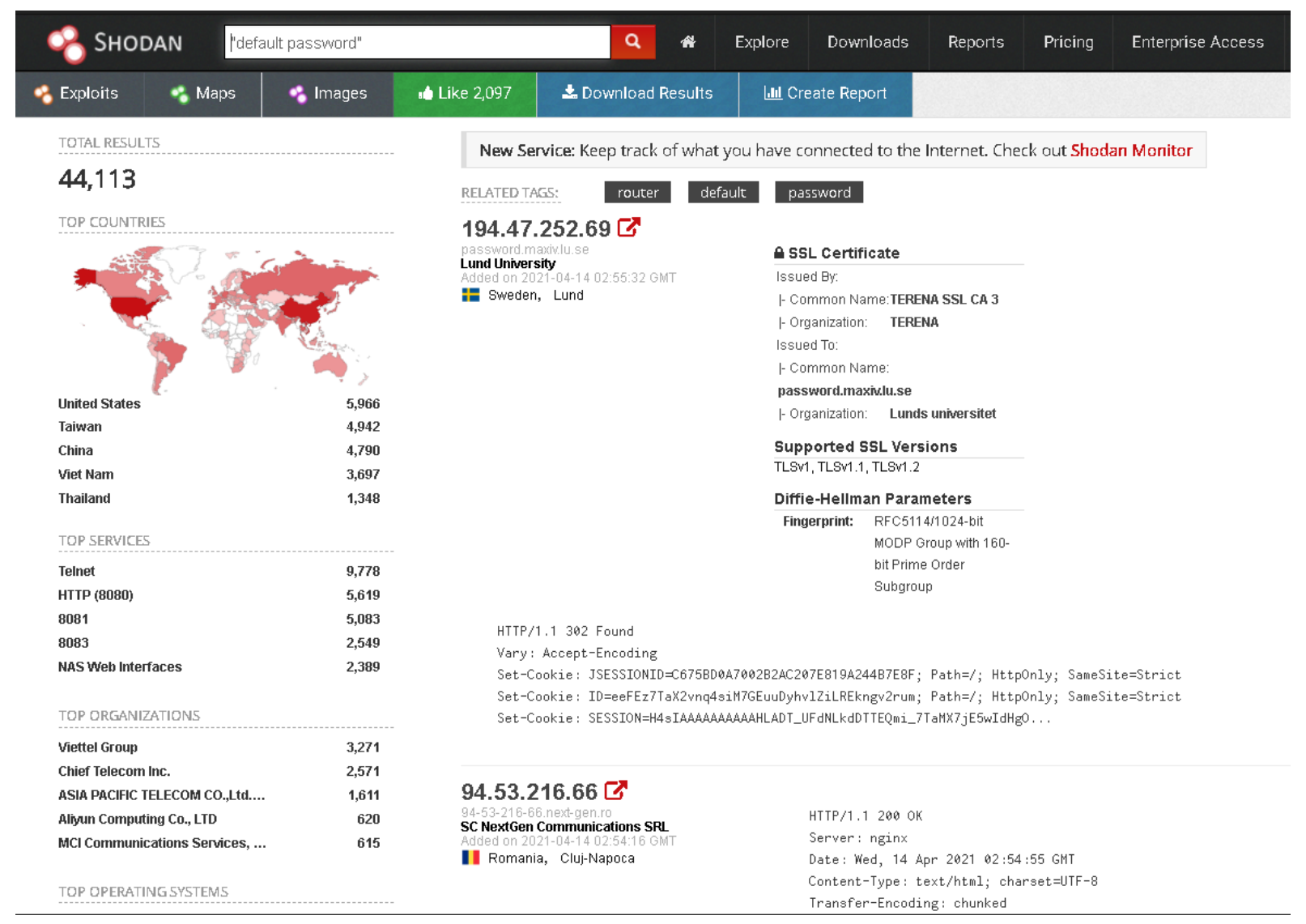

Figure 1.6: Shodan default passwords router search

\subsection{Research Questions}

The trials in this problem report make use of the IoT-23 dataset described in Section 1.2 and four machine learning algorithms. Those algorithms are the Random Forest, Support Vector Machine, Naïve Bayes, and a linear classifier with Stochastic Gradient Descent as the optimizer. The metrics used to evaluate the performance of these learners were accuracy, precision, recall, and F-1 score. The feature importance was extracted from the top learner based on the Gini impurity. More details about how the dataset was processed, the learners used here, and the metrics outlined above can be seen in the Methodology. This problem report explores the following two research questions:

RQ1: What learner performs the best in terms of classification accuracy, recall, precision, and $F_{1}$ score for this IoT traffic dataset?

RQ2: What are the features with the best predictive power for the classification task? 


\subsection{Contributions}

Although the field of IoT malware is well-studied, most publications focus on static analysis of a binary of a single malware, emulated network traffic dissection, and honeypots that harvest malware samples. Work by Antonakis et al [1] examined Mirai, Torii, and Gagfyt in detail using real and emulated IoT devices and provided the PCAPs and CSV files with columns of statistical calculations already performed on the original traces. Previous research works based on the IoT-23 dataset used all of the log files and did not perform subsampling or cross-validation [48, 43].

The contributions of this problem report are as follows:

- Subsampling was used to improve the realism of the dateset with respect to the proportions of benign and malicious traffic. Furthermore, the results are more trustworthy due to using five-fold cross-validation.

- Previous studies incorporated a combination of Random Forest, Naïve Bayes, Artificial Neural Networks, and Support Vector Machines. This research is different because the number of trees in the Random Forest is greater, but their depth is shallower, a different kernel was used in the SVM, and the Stochastic Gradient Descent was used for the first time for classification on the IoT-23 dataset in a linear classifier that is not seen in other work on this dataset or similar datasets. 


\section{Chapter 2}

\section{Related Work}

A common technique for generating malicious traffic datasets is to use a honeypot framework and collect information in log files [53]. The advantage to this technique is the potential novel exposure to new malware families and variants not seen in previous literature. Vishwakarma and Jain used a collection of honeypots in virtual machines that did not require user-input or interaction with the malware to operate, extracted features from the log files generated, and trained a variety of classifiers on the data [53]. To perform near real-time detection of malicious activity they extracted features similar to the ones from the Zeek logs in this problem report, a such as packet length, protocol, interval between packets, etc. [53]. Their primary contribution was the collection framework, which used lightweight stateless features and binary classifiers to distinguish DDoS traffic [53]. Accuracy, recall, and other metrics were not reported.

Several behavior-based approaches to malware analysis and detection used system call traces and hardware performance counters to characterize malicious activity [32, 3]. This form of dynamic analysis relies on a baseline of benign activity that can be compared against unclassified activity to determine whether it is safe. Indicators used to describe benign interactions with the operating system include the resources used by the system call, the time the action occurred, and the traces generated [3]. A disadvantage of this strategy is high false negatives that need correction [3]. Nari and Ghorbani used similar techniques to automate extraction of network features from PCAPs [32]. Some of the features they used were port number, IP address, and protocol [32]. They compared the per-class accuracy of their automated framework to the actual labeled flows from the Communication Research Center Canada dataset [32].

Hegde et al performed Big Data analysis on Mirai, Torii, Gagfyt, Kenjiro, Okiru, and 
several smaller trojans using decision trees, multi-class decision forest, Random Forest (RF), and a multi-class neural network [19]. Stoian also implemented a random forest, Naïve Bayes, Multi-Layer Perceptron (MLP), Support Vector Machine, and artificial neural network (ANN) variant using various hyper-parameters to compare them [48]. Most studies that take advantage of the IoT-23 dataset answer the question: "What are the best machine learning algorithms for detecting [or classifying] anomalies [malicious traffic] generated by IoT devices?" Multiple studies showed high accuracy (95\% or greater) with Random Forests. Garcia and Muga used this model to classify an imbalanced dataset of approximately 9,300 malware and their variants using a stratified sampling method to prevent overfitting and undergeneralization [17]. They also converted the binaries into 8-bit vectors that were plotted as grayscale images of varying sizes and patterns that were partitioned into a training and testing set using an 80:20 split and fed into their Random Forest model [17]. They used a 10-fold cross-validation to evaluate the training set and train the model [17]. The training and test sets consisted of a 1024 feature vectors and a corresponding label [17]. Their model had a 95\% accuracy and a Kappa statistical value of 94\%, indicating a strong predictive capability [17]. One of the main challenges researchers in this domain face are high rates of false positives or negatives, across several models [48]. One of the poorest performers was the SVM and Artificial Neural Network (ANN). The best performers were typically RFs and NB. 


\section{Chapter 3}

\section{Description of the dataset and features}

\subsection{IoT-23 Dataset}

The IoT-23 dataset published in 2020 by Parmisano, Garcia, and Erquiaga contains packet captures and Zeek (formerly known as Bro) logs from twenty malware and three benign traffic samples [36]. The scenarios selected are seen in Table 3.3. The authors detonated specific malware in a Raspberry $\mathrm{Pi}$, which became patient zero in the infection chain [36]. The malware samples spread to real IoT devices [36]. According to the authors, both mailicious and benign scenarios "ran in a controlled network environment with unrestrained internet connection like any other real IoT device" [36]. The IoT devices exploited were an Amazon Echo, Phillips HUE mart LED lightbulb, and Somfy smart lock [36].

The Zeek logs were obtained by running the Zeek network analyzer on the original PCAP files, which were used as the primary data source for analysis in this project [36]. Although most of the captures were conducted over a 24-hour period, some generated too much traffic to stay alive for this long. One example is the IRCBot malware, which is a trojan that uses Internet Relay Chat (IRC) servers to communicate with botmasters, giving them remote access and allows access to MSN Messenger contacts [36, 29]. Initially, the conn.log files contained the following numbers of Zeek flows by malware: Torii $(6,497)$, Hajime $(6,378,294)$, Hakai $(10,404)$, and Hide and Seek $(1,008749)$. Apart from Hide and Seek, which was collected over 112 hours, the other three were online for 24 hours. The authors also created labels to indicate the precise purpose of each packet for malicious flows [36].

The file used to aggregate the network traffic flows from the PCAP files is Zeek's conn.log. 
This log records traffic information at the Layer 3 and Layer 4 levels of the OSI model and answers the questions "who is talking to whom, in what way, and for how long?" Figure 3.1 and Figure 3.2 depict a typical packet analysis frame of tshark (the CLI component of Wireshark), with highlighted segments indicating what Zeek extracts from packets in capture files. As seen in Figure 3.3, the contents take the form of 18 key-value pairs in a JSON object.

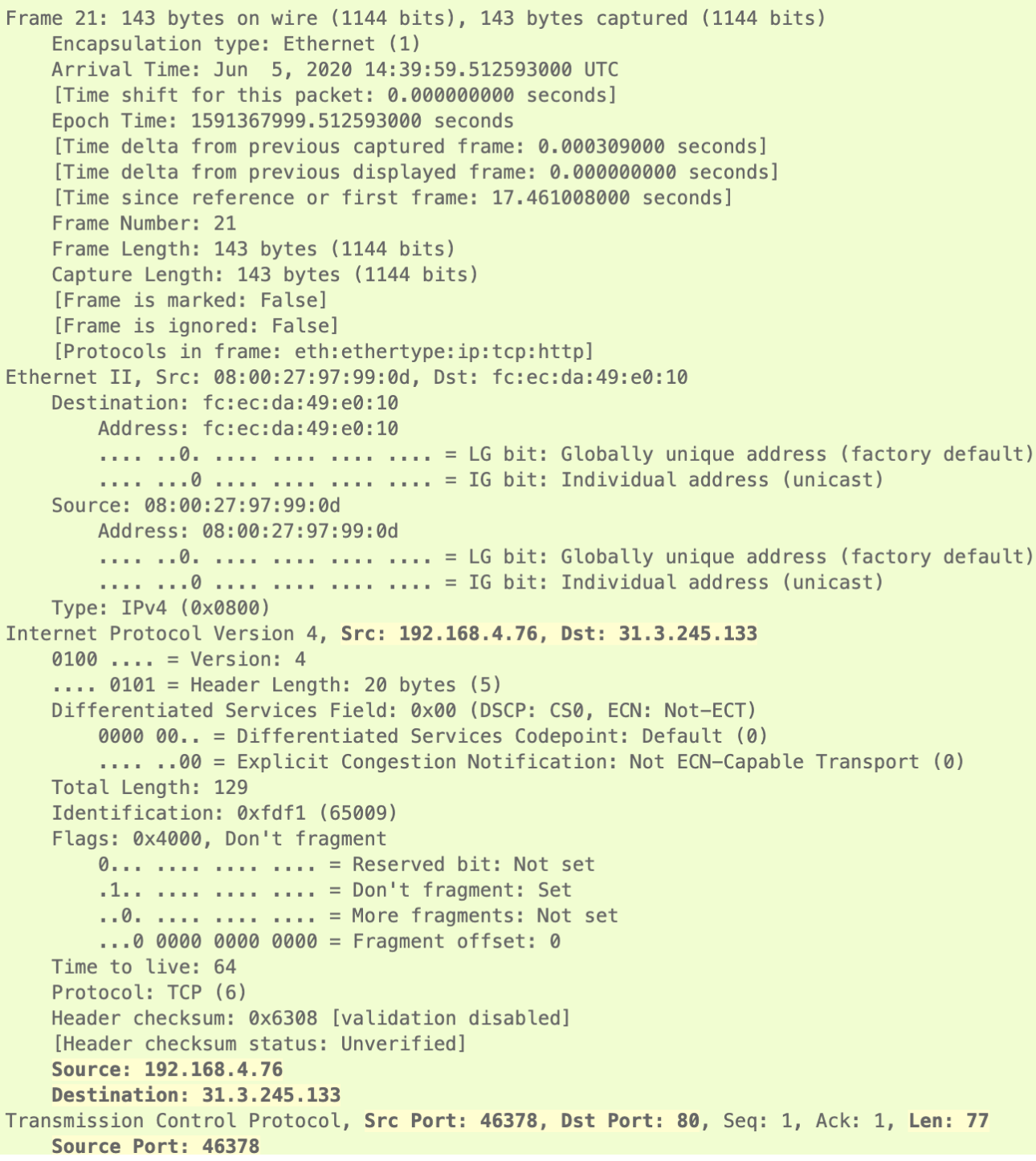

Figure 3.1: Wireshark frame with Zeek capture elements: Part 1 
$\ldots \ldots 1 \ldots=$ Acknowledgment: Set

$\ldots \ldots, \ldots=$ Push: Set

........ . .. = Reset: Not set

$\ldots \ldots \ldots . . . .0$ Syn: Not set

$\ldots \ldots \ldots 0=$ Fin: Not set

[TCP Flags: $\cdots \cdots \cdot$ AP $\cdots$ ]

Window size value: 32

[Calculated window size: 65536]

[Window size scaling factor: 2048]

Checksum: 0xd9f0 [unverified]

[Checksum Status: Unverified]

Urgent pointer: 0

Options: (12 bytes), No-Operation (NOP), No-Operation (NOP), Timestamps

TCP Option - No-Operation (NOP)

Kind: No-Operation (1)

TCP Option - No-Operation (NOP)

Kind: No-Operation (1)

TCP Option - Timestamps: TSval 3137978878, TSecr 346747623

Kind: Time Stamp Option (8)

Length: 10

Timestamp value: 3137978878

Timestamp echo reply: 346747623

[SEQ/ACK analysis]

[iRTT: 0.082118000 seconds]

[Bytes in flight: 77]

[Bytes sent since last PSH flag: 77]

[Timestamps]

[Time since first frame in this TCP stream: 0.082427000 seconds]

[Time since previous frame in this TCP stream: 0.000309000 seconds]

TCP payload (77 bytes)

Hypertext Transfer Protocol

GET / HTTP/1.1\r\n

[Expert Info (Chat/Sequence): GET / HTTP/1.1\r\n]

[GET / HTTP/1.1\r\n]

[Severity level: Chat]

[Group: Sequence]

Request Method: GET

Request URI: /

Request Version: HTTP/1.1

Host: testmyids.com $\backslash r \backslash n$

User-Agent: curl/7.47.0\r\n

Accept: $* / * \backslash r \backslash \mathrm{n}$

$\backslash \mathbf{r} \backslash \mathbf{n}$

[Full request URI: http://testmyids.com/]

[HTTP request $1 / 1$ ]

Figure 3.2: Wireshark frame with Zeek capture elements: Part 2 


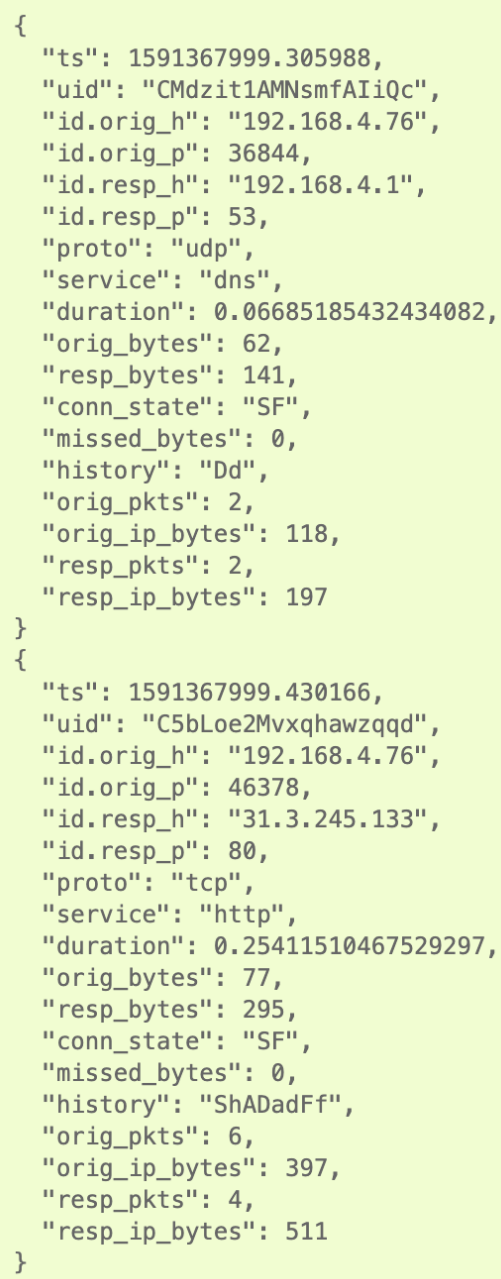

Figure 3.3: Zeek conn.log example

\subsection{Zeek Connection Log Features}

The tables below outline the Zeek conn.log features available. The columns used in this problem report are in bold in Table 3.1, while an expanded definition of the labels used in the history field is seen in Table 3.2. The original counts of packets captured and Zeek flows generated from the PCAPs in the IoT-23 dataset are seen in Table 3.3. Table 3.3 also indicates which malware ran in each scenario used. Although only three of the conn.log files explicitly indicate benign traffic in Table 3.3, there is benign traffic in all of the malicious scenarios in varying quantities. Table 3.4 indicates the definitions of detailed labels the authors provided, which are also used to illustrate the dichotomy of sample types in the descriptive results. 
Table 3.1: Zeek conn.log features (bolded features used in this problem report)

\begin{tabular}{|l|l|l|}
\hline Feature & Data Type & Description \\
\hline ts & time & Timestamp in UNIX epoch format \\
\hline uid & string & Unique ID of Connection \\
\hline id.orig_h & string & Originating endpoint's IP address (AKA ORIG) \\
\hline id.orig_p & integer & Originating endpoint's TCP/UDP port (or ICMP code) \\
\hline id.resp_h & addr & Responding endpoint's IP address (AKA RESP) \\
\hline id_resp_p & integer & Responding endpoint's TCP/UDP port (or ICMP code) \\
\hline proto & string & Transport layer protocol of connection \\
\hline service & string & Dynamically detected application protocol, if any \\
\hline duration & integer & Time of last packet seen - time of first packet seen \\
\hline orig_bytes & integer & $\begin{array}{l}\text { Originator payload bytes; from sequence numbers if } \\
\text { TCP }\end{array}$ \\
\hline resp_bytes & integer & $\begin{array}{l}\text { Responder payload bytes; from sequence numbers if } \\
\text { TCP }\end{array}$ \\
\hline conn_state & string & Connection state (see conn.log:conn_state table) \\
\hline local_orig & bool & $\begin{array}{l}\text { If conn originated locally T; if remotely F. If } \\
\text { Site::local_nets empty, always unset. }\end{array}$ \\
\hline missed_bytes & integer & Number of missing bytes in content gaps \\
\hline history & string & Connection state history (see conn.log:history table) \\
\hline orig_pkts & integer & Number of ORIG packets \\
\hline orig_ip_bytes & integer & $\begin{array}{l}\text { Number of ORIG IP bytes (via IP total_length header } \\
\text { field) }\end{array}$ \\
\hline resp_pkts & integer & Number of RESP packets \\
\hline resp_ip_bytes & integer & $\begin{array}{l}\text { Number of RESP IP bytes (via IP total_length header } \\
\text { field) }\end{array}$ \\
\hline tunnel_parents & set & If tunneled, connection UID of encapsulating parent (s) \\
\hline
\end{tabular}


Table 3.2: Zeek history reference with description

\begin{tabular}{|l|l|}
\hline History Indicator & Description \\
\hline S & A SYN without the ACK bit set \\
\hline H & SYN-ACK handshake \\
\hline A & Pure ACK \\
\hline D & Packet with data payload \\
\hline F & Packet with FIN bit set \\
\hline R & Packet with RST bit set \\
\hline C & Packet with bad checksum \\
\hline I & Inconsistent packet with both SYN and RST \\
\hline Q & Multi flag. Both SYN and FIN or SYN and RST \\
\hline T & Retransmitted packet \\
\hline N & Flipped connection \\
\hline
\end{tabular}

Table 3.3: Number of packets in original packet captures, Zeek flows, and malware in scenarios used

\begin{tabular}{|l|l|l|l|}
\hline Scenario & Malware & Packets & Zeek Flows \\
\hline CTU-IoT-Malware-Capture-1-1 & Hide N'Seek & $1,686,000$ & $1,008,749$ \\
\hline CTU-IoT-Malware-Capture-3-1 & Muhstik & 496,000 & 156,104 \\
\hline CTU-IoT-Malware-Capture-7-1 & Linux.Mirai & $11,000,000$ & $11,454,723$ \\
\hline CTU-IoT-Malware-Capture-9-1 & Linux.Hajime & $6,437,000$ & $6,378,294$ \\
\hline CTU-IoT-Malware-Capture-42-1 & Trojan & 24,000 & 4,427 \\
\hline CTU-IoT-Malware-Capture-60-1 & Gagfyt & $271,000,000$ & $3,581,029$ \\
\hline CTU-IoT-Malware-Capture-36-1 & Okiru & $13,000,000$ & $13,645,107$ \\
\hline CTU-IoT-Malware-Capture-33-1 & Kenjiro & $54,000,000$ & $54,454,592$ \\
\hline CTU-Honeypot-Capture-4-1 & Benign-Philips HUE & 21,000 & 461 \\
\hline CTU-Honeypot-Capture-5-1 & Benign-Amazon-Echo & 398,000 & 1,383 \\
\hline CTU-Honeypot-Capture-7-1 & Benign-Somfy & 8,276 & 139 \\
\hline
\end{tabular}


Table 3.4: Detailed labels of the malicious activity of each flow in the IoT-23 Zeek logs

\begin{tabular}{|c|c|}
\hline Label & Semantic Meaning \\
\hline Attack & $\begin{array}{c}\text { Attack of some sort from an infected host to a clean one. Flow } \\
\text { payload and/or behavior indicated a service was being abused } \\
\text { (e.g. telnet password brute-forcing, command injection in a GET } \\
\text { request header, etc.) }\end{array}$ \\
\hline Benign & No suspicious or malicious activity. \\
\hline $\mathrm{C} \& \mathrm{C}$ & $\begin{array}{l}\text { Infected device connected to the C\&C server. Characterized by } \\
\text { periodic connections to a malicious domain and/or download of } \\
\text { suspicious binaries. }\end{array}$ \\
\hline DDoS & $\begin{array}{l}\text { The infected host is being used in a DDoS attack. Detected } \\
\text { because the volume of traffic directed to the same IP address. }\end{array}$ \\
\hline FileDownload & Indicates a file was downloaded to the infected host. \\
\hline HeartBeat & $\begin{array}{l}\text { Packets in this connection were used to track infected hosts by } \\
\text { C\&C server. Detected by filtering connections with response bytes } \\
\text { lower than } 1 \mathrm{~B} \text { and periodic similar connections to a malicious } \\
\text { domain. }\end{array}$ \\
\hline Mirai & $\begin{array}{l}\text { Indicates these connections have characteristics of the Mirai } \\
\text { botnet family. }\end{array}$ \\
\hline Okiru & $\begin{array}{l}\text { Indicates these connections have characteristics of the Okiru } \\
\text { botnet family. }\end{array}$ \\
\hline $\begin{array}{l}\text { PartOfAHorizontal- } \\
\text { PortScan }\end{array}$ & $\begin{array}{c}\text { Connections are used to conduct port scan reconnaissance for } \\
\text { further attacks. The pattern that informed this label is the shared } \\
\text { port and similar number of transmitted bytes and multiple } \\
\text { destination IP addresses. }\end{array}$ \\
\hline Torii & $\begin{array}{l}\text { Indicates these connections have characteristics of the Torii botnet } \\
\text { family. }\end{array}$ \\
\hline
\end{tabular}




\section{Chapter 4}

\section{Machine Learning}

\subsection{Approach}

The IoT-23 dataset described in detail above was preprocessed by performing descriptive statistics and counting unique values of categorical columns, then dropping columns with mainly null values. This dataset was also extremely unbalanced in its raw form with benign traffic representing only $1 \%$ of the total connection flows. Since traffic generated by a real organization or household is the opposite, subsampling needed to occur. After preprocessing, the columns were scaled using sklearn's StandardScaler class. The supervised machine learning algorithms used in this research were Random Forest, Naïve Bayes, SVM with a linear kernel, and a linear classifier with Stochastic Gradient Descent. These models are explained in greater depth below. Training and evaluation used a five-fold cross-validation from sklearn. The performance metrics used to evaluate the models during this training and validation pipeline were accuracy, precision, recall, and F-1 score.

To avoid the perils of overfitting and selection bias, a five-fold cross-validation procedure was used to evaluate the model. In k-fold cross validation, training data is randomly partitioned into $\mathrm{k}$ different subsamples with equal sizes [17]. One subsample is held out as a test set and the remaining $\mathrm{k}-1$ subsamples are used for training [17]. This process is then repeated k-times (referred as the number of folds) with each of the $\mathrm{k}$ subsamples used as validation [17]. The resulting accuracies for each fold is averaged to produce a single estimation of the models accuracy [17].

Data analysis was performed on a Windows Server 2019 build with 64 GB of RAM running in dual-channel, an NVIDIA 2080ti GPU, and an AMD Ryzen 3950X Threadripper CPU. The Python scripts used to perform preprocessing and analysis were run in Anaconda. 
Although this setup should give abundant resources for a roughly 11 GB dataset, the columns in the Pandas dataframes needed to be downcast to their least memory intensive data types for the RAM not to be exceeded when the random forests were generated in the crossvalidation steps, as all trees are instantiated at the time of running them, which included 50 estimators (decision trees) and a depth of six nodes per tree.

\subsubsection{Random Forest}

Random Forests are a collection, or ensemble of supervised tree classifiers that each cast a unit vote for the most promising class at a given input [6, 31]. Each branch of a node or level in the tree examines a feature of the dataset and uses a threshold to separate the outputs [6]. For each tree, the features used in the starting iteration are randomly selected and the dataset is randomly sampled with replacement (bagging) during training [6]. This practice promotes diversity in the decision path of trees and combinations of features [6]. Having more variety in the trees ultimately results in clusters of high-performing trees that are able to separate datapoints into classes better than others [6]. The features in these highperforming trees become the most important and the errors of the trees with greater class impurity become less important [6]. The accuracy of the random forest classifier depends on the independence and accuracy of the individual decision tree classifiers composing it [6]. They are particularly robust against problems with high-dimensionality and always converge with greater volumes of trees, according to Breiman [6]. Random Forests are frequently used for classification of malware and malicious traffic flows. One mechanism they use for adapting to specific datasets that are unbalanced includes applying a heavy penalty to misclassification of minority classes [31].

\subsubsection{Naïve Bayes}

Naïve Bayes is a supervised learning classifier based upon the "naïve" assumption of independence among predictors (features) that produces probability distributions based upon Bayes' Theorem [39, 37]. Naïve Bayes (NB) calculates the conditional class probabilities of sample vectors from the dataset. This model is particularly well-suited to high-dimensional datasets and generally is a fast algorithm, with respect to classifiers that use kernel functions such as SVMs [37]. This model is used frequently in spam filtering and image classification due to its efficiency [30].

Disadvantages of this model include the "zero frequency" outcome if a category label not 
encountered in training is seen in the validation dataset [37]. Another issue is: features are not always independent, making this models assumption faulty [39]. The variant of NB used in this research is the Gaussian NB from sklearn, which is based on a Gaussian distribution (also called a normal distribution) of class values and is commonly used in image classification exercises or where data is continuous [8]. At each point in the dataset, the z-score distance between that point and the class mean is calculated.

\subsubsection{SVM}

An SVM is a supervised learner that generally performs well, given normalized high-dimensional data and the appropriate kernel function to avoid overfitting $[14,18,46]$. The kernel function is an algorithmic way to define a notion of similarity between points of data and transform new data such that it allows us to separate data into classes by mapping it to a different dimensional feature space. There are several main types of kernel functions: polynomial, radial, sigmoid, and linear [46]. The Linear SVM uses a linear kernel function as opposed to a radial basis function and generally takes less time to run because calculating the dot product of two points (as in the linear and polynomial kernels) is less intensive than calculating an exponential difference between a data point vector and an origin point (done in radial kernels) [15]. This trade-off in time complexity generally results in lower accuracy, but greater generalizability [14].

\subsubsection{Stochastic Gradient Descent}

Stochastic Gradient Descent (SGD) is an approach to fit linear models with convex loss functions [45]. SGD is one solution to optimization problems, which involve finding a local or global maxima or minima in a problem with multiple moving parameters [26]. In the case of a classification problem, we are minimizing the loss function and maximizing the accuracy, recall, or precision [25]. This is done by updating the parameters in the opposite direction of the gradient of the objective function (goal of maximizing or minimizing) through the learning rate for each training sample, which makes these calculations efficient because the redundancy of recalculating the gradient of similar samples is eliminated [25]. It is used successfully with large datasets and sparse machine learning problems and is typically very fast to run [45]. Typically, this technique is applied to high-dimensional datasets in natural language processing and text classification due to its efficiency and ease of application due to granular hyper-parameter control $[45,25]$. This model is highly responsive to feature scaling, 
so a standard scaler was applied to the data beforehand.

\subsection{Performance Metrics}

We introduce True Positive (TP), True Negative (TN), False Positive (FP), and False Negative (FN). True Positive in this case is a malicious flow, whereas True Negative is a benign flow. False Positive is a benign flow misclassified as a malicious flow, and False Negative is a malicious flow that is misclassified as a benign flow. Common metrics used to evaluate the performance of machine learning include accuracy, precision, recall, and F-1 score. Accuracy is defined as the fraction of correct predictions over the total number of samples or possible predictions, seen in Equation 4.1. Precision is the number of true positives divided by the number of true and false positives, seen in Equation 4.2. Recall is the fraction of instances classified towards the total sample, seen in Equation 4.3. F-1 score is the harmonic mean of precision and recall, seen in Equation 4.4.

Table 4.1: The metrics used to evaluate performance of the learners were computed from values from the confusion matrix:

\begin{tabular}{|c|c|c|}
\hline & True: Benign & True: Malicious \\
\hline Predicted: Benign & TN & FN \\
\hline Predicted: Malicious & FP & TP \\
\hline
\end{tabular}

$$
\begin{aligned}
\text { Accuracy } & =\frac{T P+T N}{T P+T N+F P+F N} \\
\text { Precision } & =\frac{T P}{T P+F P} \\
\text { Recall } & =\frac{T P}{T P+F N} \\
F_{1} & =\frac{2 \cdot \text { precision } \cdot \text { recall }}{\text { precision }+ \text { recall }}
\end{aligned}
$$

Additionally, the feature importances were calculated in the case of the Random Forest based on the Gini impurity score [44]. The Gini index measures how often a randomly 
selected row from the dataset used to train the model will be incorrectly labeled if it was randomly classified based on the distribution of labels at a given node in a decision tree [44, 6]. The Gini impurity score is used to determine which features and at what thresholds are best for splitting the data into smaller groups [44]. In an ensemble learner such as Random Forest, the average of these scores per feature is used to determine importance by ranking the features based on the total decrease in node impurity weighted by the proportion of samples reaching that node in each individual decision tree in the forest $[44,6]$.

\subsection{Pre-Processing}

The dataset utilized came from the conn.log files generated by Zeek (formerly known as Bro) after running the detection software on the original packet captures. There are typically 21 columns of possible data.

Before use, the data needed to be cleaned of null values and useless fields. The libraries used for this stage were Pandas, Sci-Kit Learn (sklearn), and Numpy. Pandas was used to read the data from the conn.log text files and coerce the objects into appropriate types so statistical models could be applied. Numpy was used to replace all '-' values that represented empty or non-existent data with NaN, which stands for "not a number" or null values. The field most affected by these replacements varied with the malware sample, making a merge of the dataframes challenging for the next stage because it is ideal to remove as many of these data points with many null values as possible. After substitution, counts of the NaN values in each column were obtained. In some cases, entire columns, such as the tunnel parents, were empty. Those fields provided no discernible value and were discarded. The service, protocol, and history features were also afflicted with NaN values to a lesser extent but were also categorical, so an average or assigned value was inappropriate. The NaN or "-" values for service, protocol, and history were treated as a separate category during numerical encoding and no alterations or row deletions were made. Some researchers who used this dataset combined all samples into one major dataset of over 325 million samples and combined the "label" and "detailed-label" fields into one super labelled column [48]. This slight compression was logical, and incorporated into the preprocessing stage for this project.

A simple numerical encoding was applied to the labels for correlation and processing. Unfortunately, sklearn does not support string categorical data in decision tree or Random Forest models. They require column data be continuous, numeric values. Before encod- 
ing the label, protocol, and service column data, the advantages and disadvantages were considered. For the typical LabelEncoder method from sklearn or the get_dummies() and category methods from Pandas, the crucial disadvantage is: the numerical encoding imposes ordinality, causing services like DHCP or DNS to be ranked, which is undesirable. One-Hot encoding converts all the possible values for the categorical data into an orthogonal vector space of with binary representation, but afflicts us with the curse of dimensionality.

Next, the values were fit and transformed using the sklearn StandardScaler class to standardize the columns using unit variance [12]. The expression for unit variance is:

$$
z=\frac{(x-u)}{s}
$$

where $\boldsymbol{x}$ is a sample, $\boldsymbol{s}$ is the standard-deviation, and $\boldsymbol{u}$ is either zero or the mean of the training sample. Centering and scaling happen independently on each feature by computing the relevant statistics on the samples in the training set, while mean and standard deviation are then stored in the transform method to be used later.

\subsection{Feature Inclusion / Exclusion Criteria}

Some of the features were immediately discarded because their values were mostly null or empty, these include the Local-Remote Connection, Connection State, and Tunnel Parent fields. Other fields such as the unique ID for each Zeek flow and IP address were not useful and could have threatened the validity of the model, such as always identifying an IP address of the malicious Raspberry Pi and any traffic emanating from it as malicious. This would produce a model with limited utility and few differences from a blacklist or whitelist. The 14 features kept were: time, origin port, respond IP address, protocol, service, duration, originator payload bytes count, responding payload bytes, missing bytes, history, ORIG packets, ORIG IP bytes, RESP packets, and RESP IP bytes. The labels were stored separately as numerically encoded categories, seen in Table 3.1. 


\section{Chapter 5}

\section{Results of Machine Learning}

\subsection{Analysis of the Dataset}

Graphs are presented below to illustrate the dichotomy of services and composition of the final dataset, seen in Figure 5.3 and Figure 5.1, respectively. It is evident this dataset is extremely unbalanced, in favor of malicious traffic. There were approximately 11 million flows, combined from $11 \log$ files of varying sizes. A population skewed in favor of malicious traffic is unrealistic, so subsampling occurred.

After subsampling, there were 1,027,714 log entries used in the trials. $50 \%$ of the rows were benign traffic, while the remaining $50 \%$ was split amongst five different types of malicious activity, seen in Figure 5.1. A breakdown of the malicious categories of traffic by detailed label is seen in Figure 5.2. Two classes of malicious activity dominated the malicious samples: DDoS attacks and port scans; with only a few hundred samples composing each of the remaining three classes.

The layer four protocol breakdown of the subsampled data in Figure 5.3 indicates a high proportion of UDP traffic, which can be partially explained by the abundance of DDoS samples, seen in Figure 5.1. DDoS activity is partially responsible because UDP and SYN floods are main approaches botnets take to conduct these attacks [24]. 
Count of Subsampled Datapoints with Detailed Label

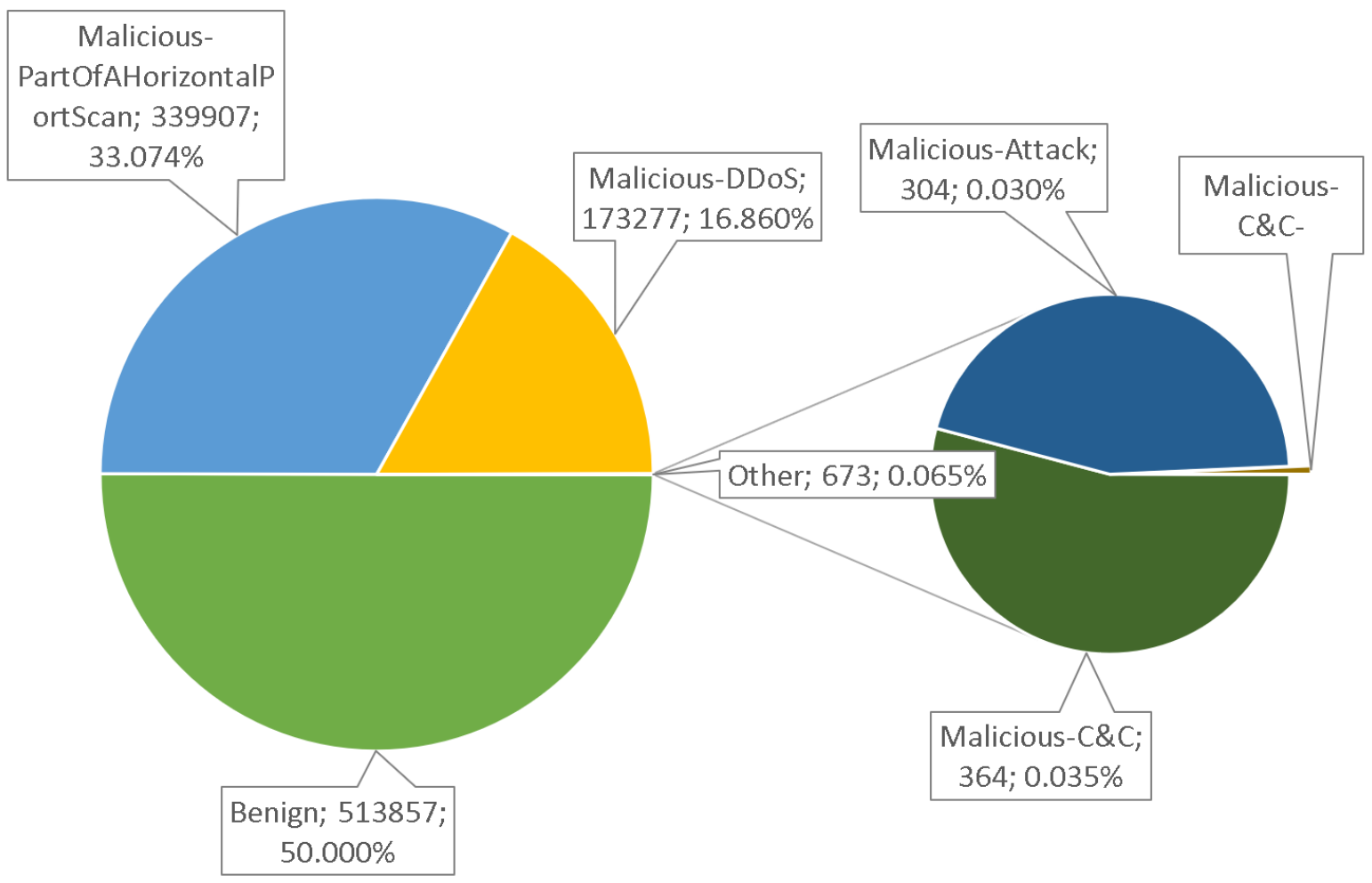

Figure 5.1: Full dataset breakdown after subsampling malicious category 


\section{Malicious Flow Counts}

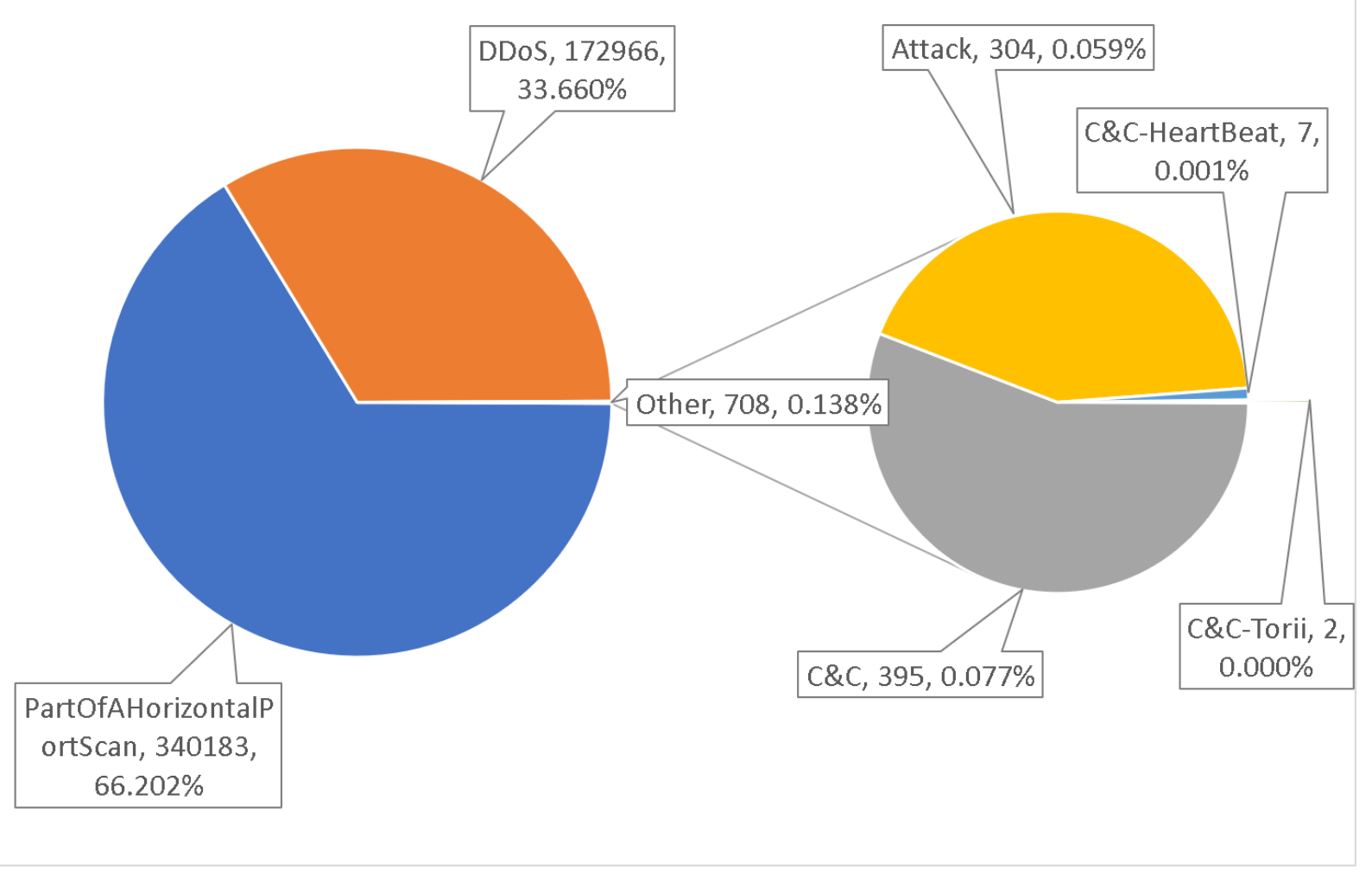

Figure 5.2: Detailed breakdown of malicious label subgroups after subsampling 
Layer 4 Protocol Representation

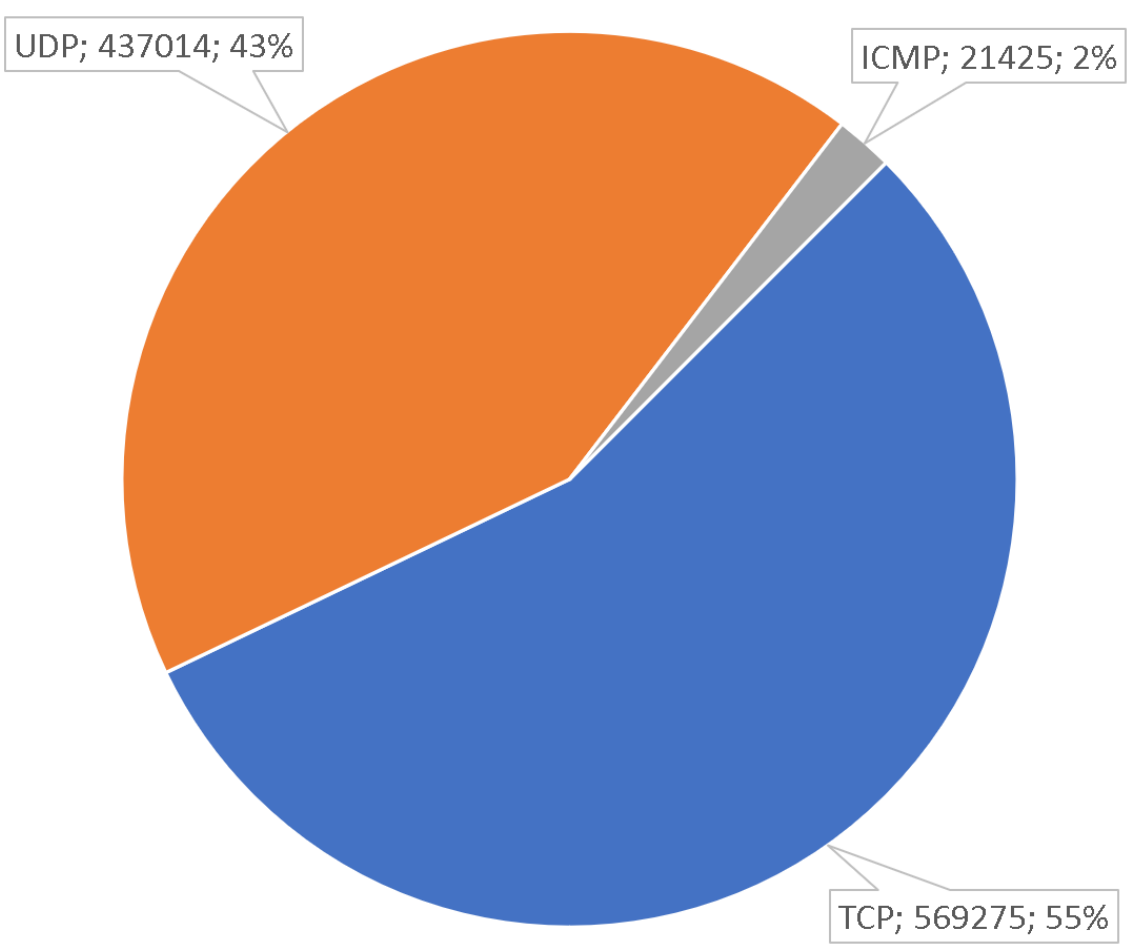

Figure 5.3: Protocol count of malicious and benign traffic

\subsection{RQ1: What learner performs the best in terms of classification accuracy, recall, and precision for this IoT traffic dataset?}

In consideration of the performance metrics described in the Methodology sections, the positive class was the malicious traffic and the negative class was the benign traffic in this problem report. The top learner in accuracy, F-1 score, and precision was the Random Forest. This strong predictive capability is likely due to the number of estimators (50 trees per forest) and the overall stability they provide. Additionally, the depth of six seems to capture the most important features well enough to split the data effectively without any further nodes. Scores are summarized in Table 5.1, 5.2, Table 5.3, Table 5.4, and all models exceeded 85\% in accuracy, recall, precision, and F-1 scores. This indicates moderate to strong predictive capability of actual malicious events. 
Linear SVM had the worst precision score, which indicates it has the highest number of false positives; however the recall score of $99.90 \%$ indicates a low rate of false negatives is promising, seen in Table 5.4 and Table 5.3. This indicates a detection model would likely misclassify benign traffic, but almost certainly catch malicious traffic that is assigned as the positive class. It is likely this occurred because the decision boundary was not adequately drawn in the model. This is typically due to poor hyperparameter tuning of the regularization parameter $(\mathrm{C})$, as this affects whether the decision boundary is smooth with greater generalizability or intricate and classifies more data points correctly at the expense of applicability to a wider population. Another explanation is: SVMs do not perform as well when there are overlapping classes or noise in the data it is possible that was the case here.

Table 5.1: Statistics of models accuracy

\begin{tabular}{|l|l|l|l|l|}
\hline Accuracy & Mean & Median & Variance & IQR \\
\hline Random Forest & $97.45 \%$ & $97.48 \%$ & $2.9996 \mathrm{E}-07$ & 0.001158 \\
\hline Naïve Bayes & $94.19 \%$ & $94.19 \%$ & $2.2552 \mathrm{E}-08$ & 0.000302 \\
\hline Linear SVM & $91.72 \%$ & $91.70 \%$ & $1.0974 \mathrm{E}-07$ & 0.000705 \\
\hline Stochastic Gradient Descent & $94.45 \%$ & $94.46 \%$ & $1.4464 \mathrm{E}-06$ & 0.002043 \\
\hline
\end{tabular}

Table 5.2: Statistics of models F-1 scores

\begin{tabular}{|l|l|l|l|l|}
\hline F-1 & Mean & Median & Variance & IQR \\
\hline Random Forest & $97.39 \%$ & $97.41 \%$ & $3.2197 \mathrm{E}-07$ & 0.001201 \\
\hline Naïve Bayes & $94.49 \%$ & $94.50 \%$ & $1.8005 \mathrm{E}-08$ & 0.000267 \\
\hline Linear SVM & $92.35 \%$ & $92.33 \%$ & $8.1935 \mathrm{E}-08$ & 0.000603 \\
\hline Stochastic Gradient Descent & $94.44 \%$ & $94.41 \%$ & $1.9727 \mathrm{E}-07$ & 0.000753 \\
\hline
\end{tabular}

Table 5.3: Statistics of models recall

\begin{tabular}{|l|l|l|l|l|}
\hline Recall & Mean & Median & Variance & IQR \\
\hline Random Forest & $94.98 \%$ & $94.98 \%$ & $6.1962 \mathrm{E}-07$ & 0.00166 \\
\hline Naïve Bayes & $99.75 \%$ & $99.75 \%$ & $1.8128 \mathrm{E}-08$ & 0.000248 \\
\hline Linear SVM & $99.90 \%$ & $99.91 \%$ & $2.6588 \mathrm{E}-08$ & 0.000248 \\
\hline Stochastic Gradient Descent & $93.51 \%$ & $93.46 \%$ & $7.3624 \mathrm{E}-07$ & 0.001815 \\
\hline
\end{tabular}


Table 5.4: Statistics of models precision

\begin{tabular}{|l|l|l|l|l|}
\hline Precision & Mean & Median & Variance & IQR \\
\hline Random Forest & $99.92 \%$ & $99.92 \%$ & $1.7082 \mathrm{E}-07$ & 0.000922 \\
\hline Naïve Bayes & $89.76 \%$ & $89.75 \%$ & $7.1658 \mathrm{E}-08$ & 0.000546 \\
\hline Linear SVM & $85.85 \%$ & $85.85 \%$ & $2.229 \mathrm{E}-07$ & 0.001038 \\
\hline Stochastic Gradient Descent & $95.33 \%$ & $95.35 \%$ & $4.6119 \mathrm{E}-07$ & 0.001341 \\
\hline
\end{tabular}

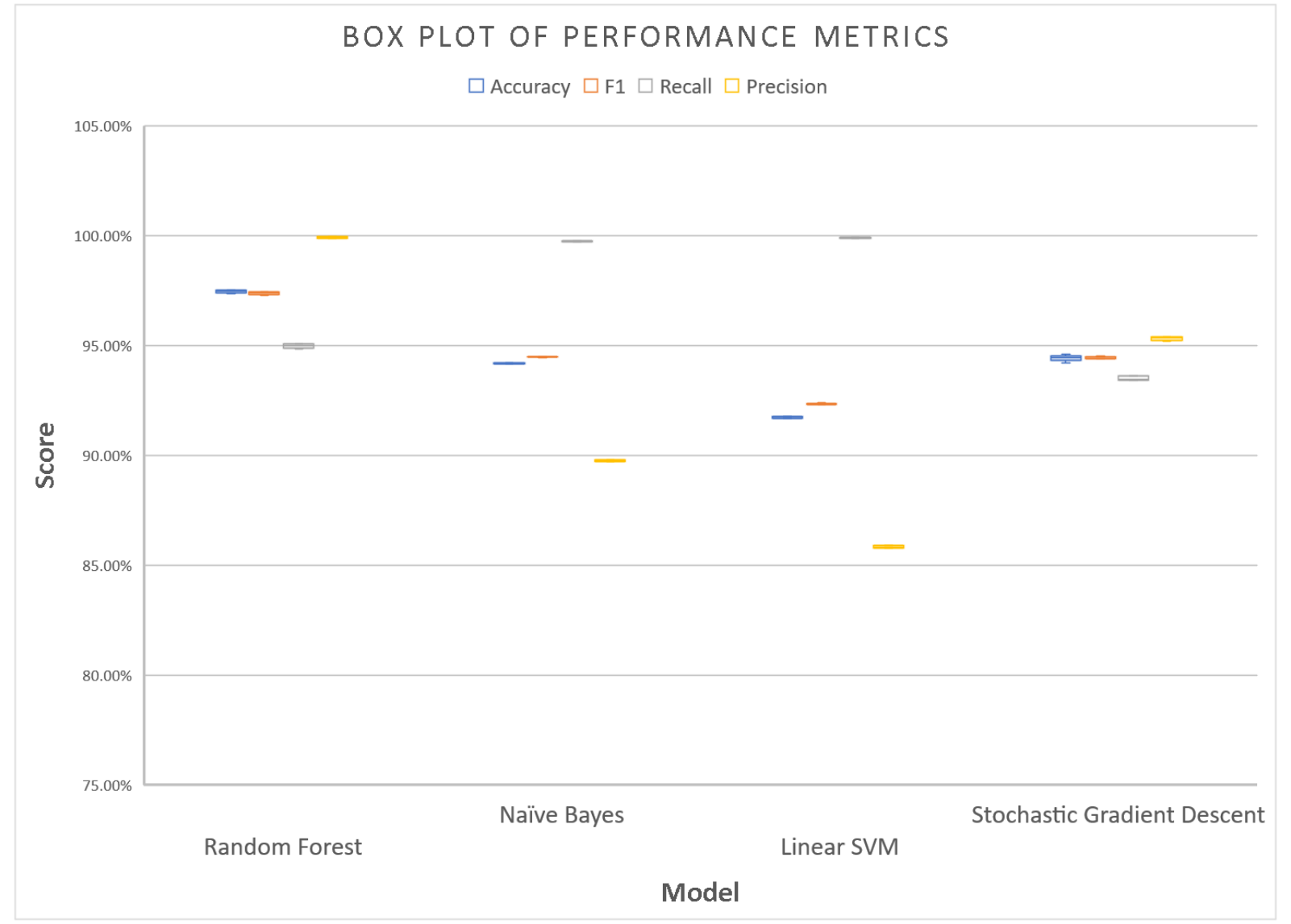

Figure 5.4: Box plot of performance metrics

\subsection{RQ2: What are the features with the best predic- tive power for classification tasks?}

To answer this question, we refer to Figure 5.5, which depicts the most important features in the Random Forest model decision trees. As stated in the Performance Metrics subsection, this ranking is based on the ability of features to adequately split data at a node better 
than randomly guessing. The responding and origin ports, count of packets from the origin, timestamp, and history of communication were the most important features. Some of these features such as volume of traffic, origin port, and time the benign or malicious action occurred are associated with typical behavior-based detection strategies $[32,3]$.

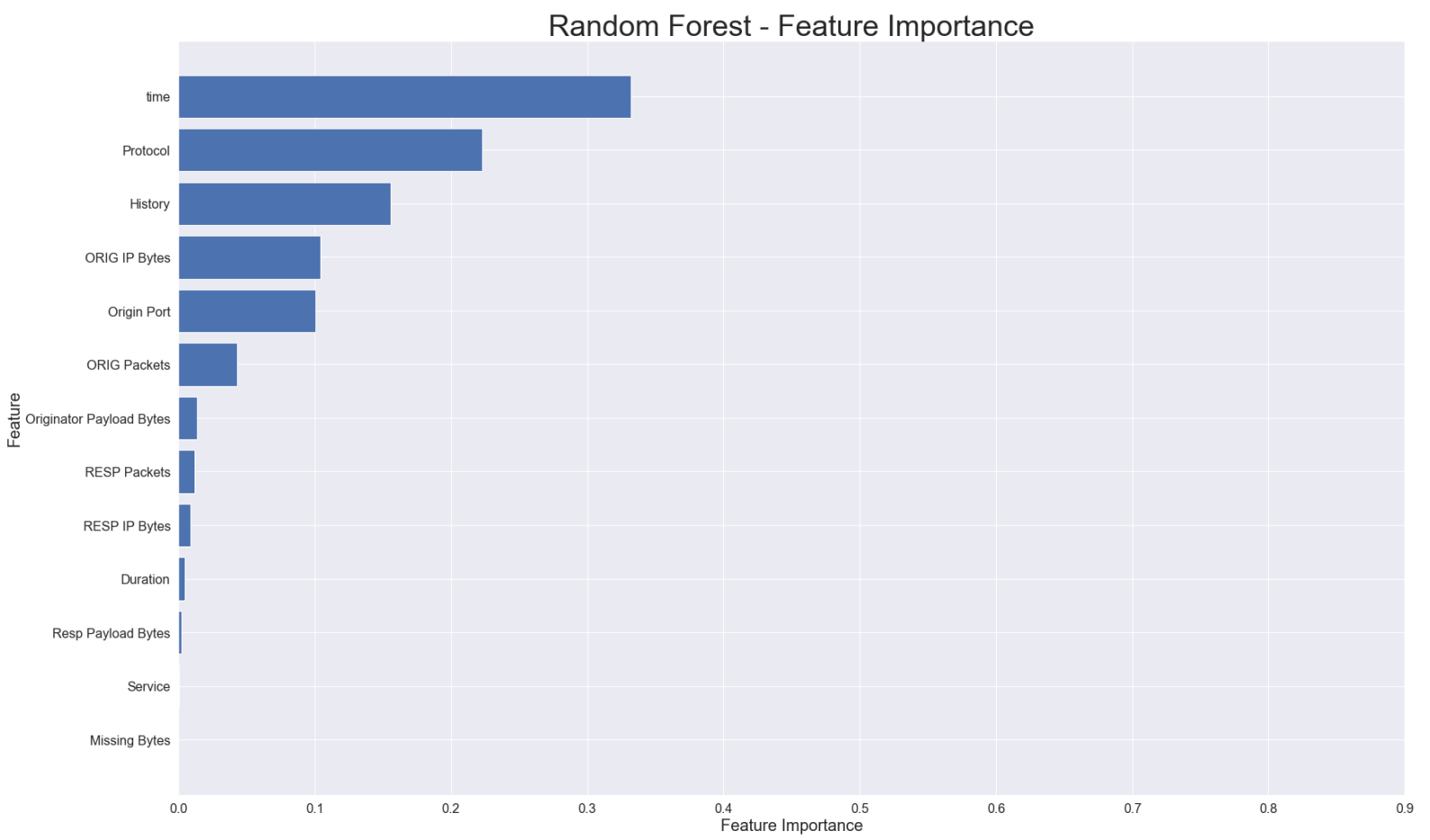

Figure 5.5: Random Forest feature importance, measured by Gini impurity 


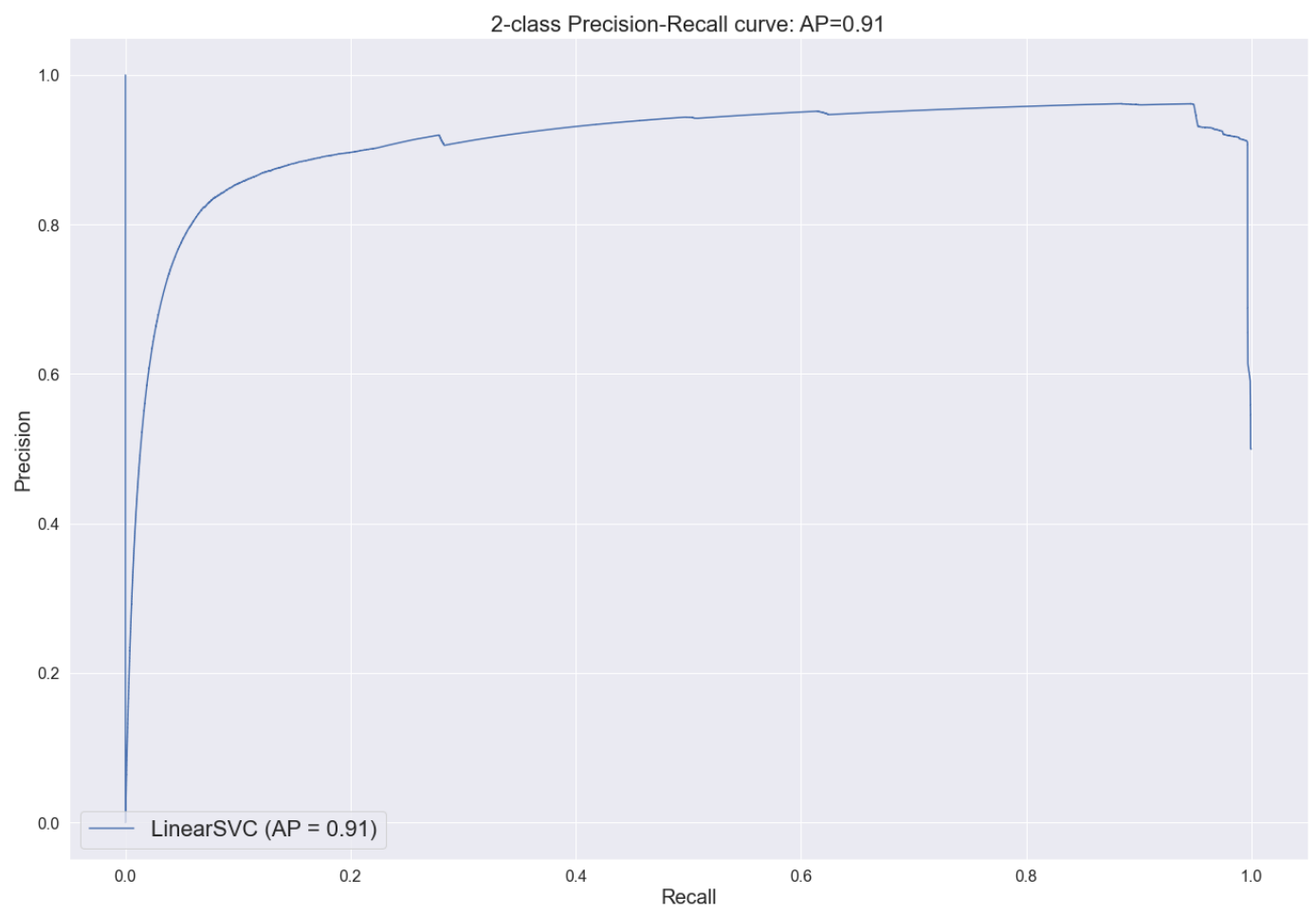

Figure 5.6: Precision-recall curve for linear SVM

\subsection{Discussion of the Main Findings}

Potential reasons the random forest and linear classifier with stochastic gradient descent algorithms performed so well can be attributed to the features deemed most important. In Figure 5.5, we see the time, history of interactions, responding IP address, and volume of packets stemming from the origin address were among the most important features. Time of day is a common indicator of malicious activity when compared to normal traffic patterns. The breakdown of malicious flows sampled, indicates the majority are port scan and DDoS activities, which would also be characterized by ICMP traffic and floods of packets in short time periods, which explains why those features were so influential in the models. 


\section{Chapter 6}

\section{Threats to Validity}

Threats to validity are environmental and structural issues that confound the results of research such that improper techniques may have been administered, leading to incorrect conclusions from data being drawn $[41,33,7]$. There are four main categories of threats to validity: internal, external, construct, and conclusion. This section is dedicated to describing how these were controlled for, and the extent to which they were controlled.

\subsection{Internal Threats to Validity}

Internal validity concerns causality of the interaction of A and B. According to Campbell, there are eight classes of extraneous variables that threaten correct interpretation of causality; however, only four apply to software: history, repeated testing, calibration of instruments. Shuffling of the dataset was also implemented prior to training the model [7]. The data frame was split in an 80:20 fashion so the model never experienced the exact flows used in the validation set during the training epochs, history is unlikely to be an issue. Although testing was run in multiple trials, the classifier was a fresh instance in each run, in both pilot and cross-validation runs. Furthermore, no weights or biases were loaded from previous trials. This leads us to believe there was no interference from previous models. Calibration of instruments in this case may refer to hyperparameters or hardware setup. The Jupyter environment and Python packages used are well-researched and documented and only attributes (hyperparameters) were modified, so it is unlikely calibration affected the accuracy, precision, or recall scores of the model. None of the devices were altered in any way by the authors, according to their descriptions of the collection environment [36]. 


\subsection{External Threats to Validity}

External validity refers to how well the results of the study can be generalized to the wider population $[7,33]$. The main issue connected to applicability outside of the experimental setup is the composition of the dataset. As seen in the descriptive environment, there was an overabundance of malicious traffic with respect to benign traffic, which is atypical for both public and private sector incident response environments. To address this imbalance, the malicious data was randomly downsampled so there were equal numbers of malicious and benign traffic flows. The devices attacked by the malware were real, instead of emulated, and the WAN connection to the internet was also unfettered, according to the original authors [36]. These give credence to the idea the training and test data were as close to a scenario found in the wild as possible, thus the results have high external validity.

\subsection{Construct Threats to Validity}

Construct validity addresses how well-suited an experimental design is to measure the theoretical concepts upon which the study is based [33]. Examples of factors that affect this are hypothesis guessing, evaluation apprehension, the manner in which missing values are addressed, and whether data collection and pre-processing adequately represent or measure the malicious or benign behavior, in this case. Another threat to the construct validity is the specific samples that were selected during preprocessing. It is recommended to perform subsampling multiple time; however, it is important to note the dataset was very large (approximately 1 million samples), so the threat is not significant.

Since real malware samples were detonated on real IoT devices that had standard outbound internet access, the traffic generated was authentic. Packet captures were made while these infected IoT devices ran for at least 24 hours and the logs Zeek generated parsed the fields directly. One issue with Zeek is: it does not always recognize the protocol used or capture all of the data such as the connection state and had the original PCAPs been used instead, more could have been distilled. Despite these shortcomings, most of the crucial protocol data and session metadata was extracted from these PCAP files, including volume of packet bytes, outbound and inbound ports and IP addresses, etc. It is unclear whether the lost data would have been beneficial or not.

The pre-processing stage had the greatest potential for construct validity issues, as almost half of the features were discarded. The columns removed were excised because they were mostly null or completely null (the connection state and tunnel parents), or their values were 
completely unique (id field that is normally used to correlate events across multiple log files).

\subsection{Conclusion Threats to Validity}

Conclusion validity pertains to the statistical measures used and the extent to which a researcher is certain the results reflect certainty in a relationship between A and B. Examples of problems that arise in this scenario are low power (tests that are not conclusive enough to reject the null hypothesis), random heterogeneity of the sample, and mistaking a trivial effect of treatment or external factors for the main influence [41]. Application of random forest and tree-based machine learning models, and the metrics used to evaluate them are wellestablished, so this is unlikely to cause problems. Furthermore, a five-fold cross validation technique was used to avoid overfitting and selection bias on the part of the model. Another potential issue is the use of Time as a discriminator for the models. Since these scenarios were run in a simulated environment for only 24 hours, the time the malware detonated may have unintentionally biased some of the results. A counterpoint is: Time, combination of software used, processes, etc. are all typical features used to identify malicious activity out of step with baseline benign behavior [32, 3]. 


\section{Chapter 7}

\section{Concluding Remarks}

In this problem report, we addressed the problem of binary classification of malicious and benign activity in IoT network traffic. The dataset consisted of 1,027,714 flows subsampled from approximately 65 million datapoints from the IoT-23 dataset generated by detonating 20 malware files on three IoT devices. The duration of each collection was 24 hours and a standard internet connection was allowed. Zeek was run on the packet captures and conn.logs were generated. The samples used in this problem report came from these conn.log files.

The results show the Random Forest to be the best performer in all categories except recall. The SVM had the greatest recall score, with Naïve Bayes as a close second. The features the Random Forest found to be most important for separating classes at the root node were time, history, protocol, responding IP address, and origin IP byte count.

The results presented in this problem report enrich the empirical evidence and explore the area of IoT malicious traffic classification in a statistically valid way. The features identified and performance of the classifiers can be used in developing lightweight, behavior-based models for detection of malicious traffic on IoT devices.

Future work would include a more diverse set of IoT devices and malware. A supermajority of the samples in this dataset were malicious, especially in the larger log files. Future studies might include more benign traffic to enhance the realism of the dataset and include additional Zeek logs such as the DNS, syslog, or files logs. With respect to machine learning, further hyper-parameter tuning and studies looking at the minimum number of trees or depth could indicate how to optimize the classification performance and resource utilization of Random Forests. Experimenting with different kernels and regularization parameters may yield more promising results for the SVM, as well. This research did not explore Adaboost or neural networks, which leaves room for innovation in this space. Since the packet captures 
are included, future research might include extraction of additional fields not parsed by Zeek. 


\section{Bibliography}

[1] Manos Antonakakis et al. "Understanding the Mirai Botnet". In: 26th USENIX Security Symposium, p. 19.

[2] Avast. New Torii Botnet uncovered, more sophisticated than Mirai - Avast. URL: https : / / blog . avast . com / new-torii-botnet-threat-research (visited on 03/16/2021).

[3] Mohammad Bagher Bahador, Mahdi Abadi, and Asghar Tajoddin. "HLMD: a signaturebased approach to hardware-level behavioral malware detection and classification". In: The Journal of Supercomputing 75 (Aug. 1, 2019). DOI: 10.1007/s11227-019-02810$\mathrm{z}$.

[4] BleepingComputer. Chinese-linked Muhstik botnet targets Oracle WebLogic, Drupal. BleepingComputer. URL: https : / / www . bleepingcomputer . com/news/security / chinese-linked-muhstik-botnet-targets-oracle-weblogic-drupal/ (visited on 04/15/2021).

[5] Bogdan BOTEZATU. New Hide 'N Seek IoT Botnet using custom-built Peer-to-Peer communication spotted in the wild - Bitdefender Labs. URL: https://labs. bitdefender. com/2018/01/new-hide-n-seek-iot-botnet-using-custom-built-peer-topeer-communication-spotted-in-the-wild/ (visited on 04/15/2021).

[6] Leo Breiman. "Random Forests". In: Machine Learning 45.1 (Oct. 1, 2001), pp. 5-32. ISSN: 1573-0565. DOI: 10.1023/A :1010933404324. URL: https://doi.org/10.1023/ A: 1010933404324 (visited on 04/01/2021).

[7] Donald T Campbell. "QUASI -EXPERIMENTAL DESIGN". In: International encyclopedia of the social sciences 5 (), p. 4.

[8] T. F. Chan, G. H. Golub, and R. J. LeVeque. "Updating Formulae and a Pairwise Algorithm for Computing Sample Variances". In: COMPSTAT 1982 5th Symposium held at Toulouse 1982. Ed. by H. Caussinus, P. Ettinger, and R. Tomassone. Heidelberg: 
Physica-Verlag HD, 1982, pp. 30-41. ISBN: 978-3-642-51461-6. DOI: 10.1007/978-3642-51461-6_3. URL: http://link. springer.com/10.1007/978-3-642-51461-6_3 (visited on 04/16/2021).

[9] Catalin Cimpanu. New Hakai IoT botnet takes aim at D-Link, Huawei, and Realtek routers. ZDNet. URL: https : //www .zdnet.com/article/new-hakai-iot-botnettakes-aim-at-d-link-huawei-and-realtek-routers/ (visited on 04/13/2021).

[10] Andrei Costin, Jonas Zaddach, and Sophia Antipolis. "IoT Malware: Comprehensive Survey, Analysis Framework and Case Studies". In: BlackHat USA (), p. 9.

[11] C. J. D’Orazio, K. R. Choo, and L. T. Yang. "Data Exfiltration From Internet of Things Devices: iOS Devices as Case Studies". In: IEEE Internet of Things Journal 4.2 (Apr. 2017). Conference Name: IEEE Internet of Things Journal, pp. 524-535. ISSN: 2327-4662. DOI: 10.1109/JIOT . 2016.2569094.

[12] David Cournapeau et al. sklearn.preprocessing.StandardScaler - scikit-learn 0.24.1 documentation. scikit-learn. 2010. URL: https : / / scikit-learn . org / stable / modules/generated/sklearn . preprocessing. StandardScaler.html (visited on 04/01/2021).

[13] CVE Details. CVE-2018-10561: An issue was discovered on Dasan GPON home routers. It is possible to bypass authentication simply by appending "?i. URL: https: / / www . cvedetails . com / cve / CVE-2018-10561/ ?q=cve-2018-10561 (visited on 03/16/2021).

[14] Glenn Fung and Olvi L. Mangasarian. "Data selection for support vector machine classifiers". In: Proceedings of the sixth ACM SIGKDD international conference on Knowledge discovery and data mining - KDD '00. the sixth ACM SIGKDD international conference. Boston, Massachusetts, United States: ACM Press, 2000, pp. 64-70. ISBN: 978-1-58113-233-5. DOI: 10.1145/347090 .347105. URL: http://portal . acm. org/citation. cfm?doid=347090 347105 (visited on 04/13/2021).

[15] Glenn Fung, Olvi L. Mangasarian, and Jude Shavlik. "Knowledge-Based Support Vector Machine Classifiers". In: In Advances in Neural Information Processing Systems 14. MIT Press, 2002, pp. 01-09.

[16] Steven Furnell et al. "Understanding the full cost of cyber security breaches". In: Computer Fraud \& Security 2020.12 (Dec. 1, 2020), pp. 6-12. ISSN: 1361-3723. DOI: 10 . 1016 / S1361-3723 (20 ) 30127 - 5. URL: https : / / wWw . sciencedirect . com / science/article/pii/S1361372320301275 (visited on 04/20/2021). 
[17] Felan Carlo C Garcia and Felix P Muga Ii. "Random Forest for Malware Classification". In: arXiv preprint arXiv:1609.07770 (), p. 4.

[18] S. Ghosh, A. Dasgupta, and A. Swetapadma. "A Study on Support Vector Machine based Linear and Non-Linear Pattern Classification”. In: 2019 International Conference on Intelligent Sustainable Systems (ICISS). 2019 International Conference on Intelligent Sustainable Systems (ICISS). Feb. 2019, pp. 24-28. DOI: 10.1109/ISS1. 2019.8908018.

[19] M. Hegde et al. "Identification of Botnet Activity in IoT Network Traffic Using Machine Learning". In: 2020 International Conference on Intelligent Data Science Technologies and Applications (IDSTA). 2020 International Conference on Intelligent Data Science Technologies and Applications (IDSTA). Oct. 2020, pp. 21-27. DOI: 10.1109/ IDSTA50958.2020.9264143.

[20] Stephen Herwig et al. "Measurement and Analysis of Hajime, a Peer-to-peer IoT Botnet". In: Proceedings 2019 Network and Distributed System Security Symposium. Network and Distributed System Security Symposium. San Diego, CA: Internet Society, 2019. ISBN: 978-1-891562-55-6. DOI: 10 .14722/ndss . 2019 . 23488. URL: https : / / www . ndss - symposium . org / wp - content / uploads / 2019 / 02 / ndss2019_02B 3_Herwig_paper.pdf (visited on 03/16/2021).

[21] Stephen Hilt. "Worm War: The Botnet Battle for IoT Territory". In: documents.trendmicro.com (), p. 30 .

[22] Fu-Hau Hsu et al. Detecting Web-Based Botnets Using Bot Communication Traffic Features. Security and Communication Networks. ISSN: 1939-0114 Pages: e5960307 Publisher: Hindawi Volume: 2017. Dec. 3, 2017. DoI: https ://doi .org/10 . 1155/ 2017 / 5960307. URL: https : / / www . hindawi . com/ journals / scn/2017 / $5960307 /$ (visited on 11/20/2020).

[23] International Telecommunication Union. Internet of Things. ITU. URL: https://www . itu . int : 443/en/ITU-T/techwatch/Pages/internetofthings . aspx (visited on $03 / 11 / 2021)$.

[24] Luis Eduardo Suástegui Jaramillo. "Malware Detection and Mitigation Techniques: Lessons Learned from Mirai DDOS Attack". In: Journal of Information Systems Engineering \&6 Management 3.3 (July 16, 2018). ISSN: 24684376. DOI: 10.20897/jisem/ 2655. URL: http : //www . jisem-journal . com/article/malware-detection-and- 
mitigation-techniques-lessons-learned-from-mirai-ddos-attack (visited on $04 / 21 / 2021)$.

[25] Nikhil Ketkar. Deep Learning with Python. Berkeley, CA: Apress, 2017. ISBN: 978-14842-2766-4. DOI: 10.1007/978-1-4842-2766-4. URL: http://link. springer.com/ 10.1007/978-1-4842-2766-4 (visited on 04/13/2021).

[26] Jayanth Koushik and Hiroaki Hayashi. "IMPROVING STOCHASTIC GRADIENT DESCENT WITH FEEDBACK". In: (2017), p. 9.

[27] Clemens Scott Kruse et al. "Cybersecurity in healthcare: A systematic review of modern threats and trends". In: Technology and Health Care 25.1 (Feb. 21, 2017), pp. 1-10. ISSN: 09287329, 18787401. DOI: 10 . 3233/THC-161263. URL: https : / www . medra. org/servlet/aliasResolver?alias=iospress\&doi=10.3233/THC-161263 (visited on $04 / 20 / 2021)$.

[28] Avast Labs. Hide 'N Seek Botnet expands - Avast. URL: https://blog.avast.com/ hide-n-seek-botnet-continues (visited on 04/15/2021).

[29] Microsoft. Backdoor:Win32/IRCbot threat description - Microsoft Security Intelligence. URL: https: //www . microsoft. com/en-us/wdsi/threats/malware-encyclopediadescription?Name=Backdoor : Win32/IRCbot (visited on 03/16/2021).

[30] Ronei Marcos De Moraes and Liliane Dos Santos Machado. Gaussian Naive Bayes for Online Training Assessment in Virtual Reality-Based Simulators.

[31] C. D. Morales-Molina et al. "Methodology for Malware Classification using a Random Forest Classifier". In: 2018 IEEE International Autumn Meeting on Power, Electronics and Computing (ROPEC). 2018 IEEE International Autumn Meeting on Power, Electronics and Computing (ROPEC). ISSN: 2573-0770. Nov. 2018, pp. 1-6. DOI: 10.1109/ROPEC. 2018.8661441.

[32] Saeed Nari and Ali A. Ghorbani. "Automated malware classification based on network behavior". In: 2013 International Conference on Computing, Networking and Communications (ICNC). 2013 International Conference on Computing, Networking and Communications (ICNC). Jan. 2013, pp. 642-647. DOI: 10.1109/ICCNC.2013.6504162.

[33] Amadeu Anderlin Neto and Tayana Conte. "A conceptual model to address threats to validity in controlled experiments". In: Proceedings of the 17th International Conference on Evaluation and Assessment in Software Engineering - EASE '13. the 17th International Conference. Porto de Galinhas, Brazil: ACM Press, 2013, p. 82. IsBN: 
978-1-4503-1848-8. DOI: 10 . 1145 / 2460999 . 2461011. URL: http : / / dl . acm . org / citation. cfm?doid=2460999 2461011 (visited on 04/06/2021).

[34] NIST. NVD - CVE-2017-17215. URL: https://nvd.nist.gov/vuln/detail/CVE2017-17215\#vulnCurrentDescriptionTitle (visited on 04/13/2021).

[35] Palo Alto Networks. Unit 42 Finds New Mirai and Gafgyt IoT/Linux Botnet Campaigns. Unit42. July 20, 2018. URL: https : / / unit42 . paloaltonetworks . com / unit42 - finds - new - mirai - gafgyt - iotlinux - botnet - campaigns/ (visited on $04 / 13 / 2021)$.

[36] Agustin Parmisano, Sebastian Garcia, and Maria Jose-Erquiaga. IoT-23 Dataset: A labeled dataset of Malware and Benign Io T Traffic. URL: https://www . stratosphereips . org/datasets-iot23 (visited on 03/12/2021).

[37] Konrad Rieck, Patrick Stewin, and Jean-Pierre Seifert, eds. Detection of Intrusions and Malware, and Vulnerability Assessment. Red. by David Hutchison et al. Vol. 7967. Lecture Notes in Computer Science. Berlin, Heidelberg: Springer Berlin Heidelberg, 2013. ISBN: 978-3-642-39235-1. DOI: 10 . $1007 / 978-3-642-39235-1$. URL: http : //link.springer.com/10.1007/978-3-642-39235-1 (visited on 04/07/2021).

[38] Rootkiter. HNS Botnet Recent Activities. 360 Netlab Blog - Network Security Research Lab at 360. July 6, 2018. URL: https ://blog.netlab.360 .com/hns-botnet-recentactivities-en/ (visited on 04/15/2021).

[39] Mucahid Mustafa Saritas and Ali Yasar. "Performance Analysis of ANN and Naive Bayes Classification Algorithm for Data Classification". In: International Journal of Intelligent Systems and Applications in Engineering 7.2 (June 30, 2019). Number: 2, pp. 88-91. ISSN: 2147-6799. DOI: 10 . $18201 /$ /ijisae. 2019252786. URL: https: //ijisae.org/IJISAE/article/view/934 (visited on 04/07/2021).

[40] Anthony Spadafora September 04 and 2018. Hakai IoT botnet infects popular router brands. ITProPortal. URL: https : / / www . itproportal . com / news / hakai - iot botnet-infects-popular-router-brands/ (visited on 04/13/2021).

[41] William R. Shadish, Thomas D. Cook, and Donald T. Campbell. Experimental and quasi-experimental designs for generalized causal inference. Boston: Houghton Mifflin, 2001. 623 pp. ISBN: 978-0-395-61556-0.

[42] Shodan. Shodan. URL: https://www.shodan.io/ (visited on 04/14/2021). 
[43] Arashpreet Singh. "USE OF MACHINE LEARNING FOR SECURING IoT". In: (), p. 10.

[44] Sonia Singh and Priyanka Gupta. Comparative Study Id3, Cart and C4.5 Decision Tree Algorithm: A Survey.

[45] sklearn. 1.5. Stochastic Gradient Descent - scikit-learn 0.24.1 documentation. URL: https://scikit-learn.org/stable/modules/sgd.html (visited on 04/13/2021).

[46] sklearn. sklearn.svm.LinearSVC - scikit-learn 0.24.1 documentation. URL: https : //scikit-learn.org/stable/modules/generated/sklearn.svm.LinearSVC.html (visited on 04/07/2021).

[47] Zhanna Malekos Smith, Eugenia Lostri, and James A Lewis. The Hidden Costs of Cybercrime. McAfee, p. 38.

[48] Nicolas-Alin Stoian. "Machine Learning for Anomaly Detection in IoT networks: Malware analysis on the IoT-23 Data set". In: Bachelor's thesis, University of Twente (), p. 10.

[49] TrendMicro. Bashlite Updated with Mining and Backdoor Commands. Trend Micro. Section: research. Apr. 3, 2019. URL: https : / / www . trendmicro . com / en_ us / research/19/d/bashlite-iot-malware-updated-with-mining-and-backdoorcommands-targets-wemo-devices.html (visited on 04/14/2021).

[50] TrendMicro. ThinkPHP Vulnerability Abused by Botnets. Trend Micro. Section: research. Jan. 25, 2019. URL: https://www.trendmicro.com/en_us/research/19/a/ thinkphp-vulnerability-abused-by-botnets-hakai-and-yowai.html (visited on 04/13/2021).

[51] International Telecommunication Union. About ITU. ITU. URL: https : //www . itu . int: 443/en/about/Pages/default.aspx (visited on 03/11/2021).

[52] Anand Ravindra Vishwakarma. "Network Traffic Based Botnet Detection Using Machine Learning". In: SJSU Master's Projects 917 (), p. 67.

[53] Ruchi Vishwakarma and Ankit Kumar Jain. "A Honeypot with Machine Learning based Detection Framework for defending IoT based Botnet DDoS Attacks". In: 2019 3rd International Conference on Trends in Electronics and Informatics (ICOEI). 2019 3rd International Conference on Trends in Electronics and Informatics (ICOEI). Apr. 2019, pp. 1019-1024. DOI: 10.1109/ICOEI. 2019.8862720. 
[54] Felix Wortmann and Kristina Flüchter. "Internet of Things: Technology and Value Added". In: Business 83 Information Systems Engineering 57.3 (June 2015), pp. 221224. ISSN: 2363-7005, 1867-0202. DOI: 10 . 1007 /s12599-015-0383-3. URL: http : //link. springer.com/10.1007/s12599-015-0383-3 (visited on 03/11/2021). 